\title{
Mechanism for the Singlet to Triplet Superconductivity Crossover in Quasi-One-Dimensional Organic Conductors
}

\author{
Kazuto Kajiwara, Masahisa TsuchiIzU, Yoshikazu Suzumura, and Claude Bourbonnais ${ }^{1}$ \\ Department of Physics, Nagoya University, Nagoya 464-8602 \\ ${ }^{1}$ Départment de Physique, Université de Sherbrooke, Sherbrooke, Québec J1K-2R1, Canada
}

(Received June 11, 2009; published September 25, 2009 in J. Phys. Soc. Jpn. 78 (2009) 104702)

\begin{abstract}
Superconductivity of quasi-one-dimensional organic conductors with a quarter-filled band is investigated using the two-loop renormalization group approach to the extended Hubbard model for which both the single electron hopping $t_{\perp}$ and the repulsive interaction $V_{\perp}$ perpendicular to the chains are included. For a four-patches Fermi surface with deviations to perfect nesting, we calculate the response functions for the dominant fluctuations and possible superconducting states. By increasing $V_{\perp}$, it is shown that a $d$-wave (singlet) to $f$-wave (triplet) superconducting state crossover occurs, and is followed by a vanishing spin gap. Furthermore, we study the influence of a magnetic field through the Zeeman coupling, from which a triplet superconducting state is found to emerge.
\end{abstract}

KEYWORDS: singlet superconductivity, triplet superconductivity, organic conductors, extendedHubbard model, renormalization group, quarter-filled, nesting deviations, Zeeman field

\section{Introduction}

Superconductivity in quasi-one-dimensional (quasi-1D) organic conductor, (TMTSF) ${ }_{2} X$, has been studied extensively in the conditions where charge and spin fluctuations play an important role due to the low dimensionality of the Fermi surface. ${ }^{1,2)}$ The possibility for triplet state superconductivity, besides the singlet one, is an issue of current interest in these materials. ${ }^{3-6)}$ From recent NMR measurements on (TMTSF $)_{2} \mathrm{ClO}_{4}$, it has been suggested that spin-triplet superconductivity may emerge out of a singlet state under magnetic field. This can occur as a field-induced phase transition that can compete with a FFLO state under strong magnetic field ${ }^{5)}$ It is important for such a study to take into account the influence of low-dimensional fluctuations due to the strongly anisotropic band structure of (TMTSF $)_{2} X$. The existence of spin fluctuations is supported by the NMR experiments in the normal phase of these materials. ${ }^{5,7)}$

Several theoretical works have been devoted to the fieldinduced phase transition to the triplet superconducting (SC) state. Shimahara ${ }^{8,9)}$ pointed out such a transition using the pairing interactions mediated by spin fluctuations, which have both components for the spin-singlet and triplet pairings. Combining mean-field and RPA methods, Belmechri et $a l .{ }^{10,11)}$ have shown that under field a singlet-triplet superconducting transition is possible, along with the occurrence of a FFLO state at intermediate strength of the magnetic field. A similar transition has been shown to occur by Aizawa et $a l .,{ }^{12,13)}$ using the RPA method for the extended Hubbard model that includes intersite repulsive interaction for longitudinal and transverse directions along the chains, and the Zeeman coupling of spins to a finite magnetic field. As shown for the Hubbard model with on-site repulsive interaction, the RPA approach, ${ }^{14-16)}$ which sums up a higher order of perturbation for electron interactions, suggests the importance of the pairing interactions mediated by spin fluctuations. Thus it is of interest to further examine fluctuations of both density waves and superconducting pairings, where the spin gap is essential to the existence of a singlet superconducting state.

These features can be properly taken into account by the renormalization group (RG) method. ${ }^{17,18)}$ The effect of a magnetic field on low-dimensional systems has been studied by the RG method mainly for the one-dimensional cases. ${ }^{19,20 \text { ) }}$ Noticeable progress has been achieved in studying superconductivity in zero field for the case of quasi-1D systems with many chains. ${ }^{21-23)}$ However, the different mechanisms by which triplet superconductivity can be stabilized in such systems, especially in finite magnetic field, have not been fully investigated within the RG scheme.

In the present work, we use the RG method up to the twoloop level ${ }^{24)}$ to study the competition between the $d$-wave singlet SC state ( $\mathrm{SC} d$ ) and the $f$-wave triplet SC state (SC $f$ ) in quasi-1D systems with interchain electron hopping and repulsive interactions. It is demonstrated that superconductivity is driven by the interplay of interchain interaction and the nesting deviations. The crossover from the SCd state to the $\mathrm{SC} f$ state occurs with increasing the interchain repulsive interaction in the presence of nesting deviations. The effect that a Zeeman coupling to the magnetic field can have on the stability of the SCd state and the emergence of a triplet SC $f$ state is also studied in details. In $\S 2$, we give the formulation of the RG technique for a many-chains quasi-1D system at quarter-filling, in the presence of magnetic field and nesting deviations. Using a four-patches decomposition of the Fermi surface, we derive the flow equations for the SCd and $\mathrm{SC} f$ response functions in the superconductivity channel, and for the spin-density-wave (SDW) and charge-density-wave (CDW) responses in the staggered density-wave channel. In $\S 3$, the results for the possible states as a function of interchain Coulomb interaction, nesting deviations and magnetic field are presented. The conditions for the stability of the SC $f$ triplet state in the calculated phase diagrams are given. Summary and discussion are presented in $\S 4$.

\section{Formulation}

\subsection{Model}

In order to study the superconductivity for the (TMTSF) ${ }_{2} X$ salt, we consider the quasi-1D extended Hubbard model, 
(a)

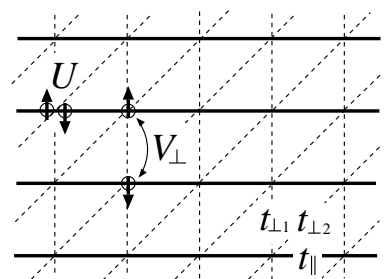

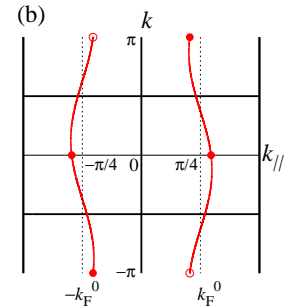

Fig. 1. (Color online) (a) Extended Hubbard model with intra- and interchain repulsive interactions. Interchain hoppings are shown by dashed lines. (b) The quasi-1D Fermi surface. The locations of the patches, 4 in number, are shown by the closed circles.

given by

$$
H=H_{0}+H_{\mathrm{I}},
$$

where

$$
\begin{aligned}
H_{0} & =-t_{\|} \sum_{j, l, \sigma}\left(c_{j, l, \sigma}^{\dagger} c_{j+1, l, \sigma}+\text { H.c. }\right) \\
& -t_{\perp 1} \sum_{j, l, \sigma}\left(c_{j, l, \sigma}^{\dagger} c_{j, l+1, \sigma}+\text { H.c. }\right) \\
& -t_{\perp 2} \sum_{j, l, \sigma}\left(c_{j, l, \sigma}^{\dagger} c_{j+1, l+1, \sigma}+\text { H.c. }\right) \\
& -\mu \sum_{j, l, \sigma} c_{j, l, \sigma}^{\dagger} c_{j, l, \sigma}-\frac{h}{2}\left(n_{j, l, \uparrow}-n_{j, l, \downarrow}\right), \\
H_{\mathrm{I}} & =U \sum_{j, l} n_{j, l, \uparrow} n_{j, l, \downarrow}+V_{\perp} \sum_{j, l} n_{j, l} n_{j, l+1} .
\end{aligned}
$$

Here $c_{j, l, \sigma}\left(c_{j, l, \sigma}^{\dagger}\right)$ is the annihilation (creation) operator of an electron at the site $j$ and chain $l$, with spin $\sigma(\sigma=\uparrow, \downarrow)$, and $n_{j, l, \sigma}=c_{j, l, \sigma}^{\dagger} c_{j, l, \sigma}$. As illustrated in Fig. 1 (a), the quantities $t_{\|}, t_{\perp 1}, t_{\perp 2}, h, \mu, U$ and $V_{\perp}$ are in order the nearest-neighbor intrachain and interchain hoppings, next-to-nearest-neighbor interchain hopping, Zeeman field $\left(\mu_{\mathrm{B}}=1\right)$, chemical potential, and finally the on-site and interchain repulsive interactions.

Equation (1) is rewritten by making use of the Fourier transform, $c_{j, l, \sigma}=\left(L N_{\perp}\right)^{-1 / 2} \sum_{\boldsymbol{k}} e^{i k_{\|} j+i k l} c_{\sigma}(\boldsymbol{k})$, where the lattice constant is taken as unity. The kinetic energy, eq. (2) can then be written as $H_{0}=\sum_{\boldsymbol{k}, \sigma} \varepsilon(\boldsymbol{k}) c_{\sigma}^{\dagger}(\boldsymbol{k}) c_{\sigma}(\boldsymbol{k})$. Here $N_{\perp}$ is the number of chains, $L$ is their length, $\boldsymbol{k}=\left(k_{\|}, k\right)$ and $\varepsilon(\boldsymbol{k})=$ $-2 t_{\|} \cos k_{\|}-2 t_{\perp 1} \cos k-2 t_{\perp 2} \cos \left(k_{\|}+k\right)$. In the quasi-1D case, we have the open Fermi surface since $\left|t_{\perp 1}\right|,\left|t_{\perp 2}\right| \ll t_{\|}$. The Fermi surface, which is a function of $k$, is divided into two parts for right-going $(p=+)$ and left-going electrons $(p=-)$ [see Fig. 1 (b)]. Further by adopting the linear-dispersion relation, eq. (2) is rewritten as

$$
\begin{aligned}
H_{0} & =\sum_{\boldsymbol{k}, \sigma} \varepsilon_{p, \sigma}(\boldsymbol{k}) c_{p, \sigma}^{\dagger}(\boldsymbol{k}) c_{p, \sigma}(\boldsymbol{k}), \\
\varepsilon_{p, \sigma}(\boldsymbol{k}) & =v\left[p k_{\|}-k_{\mathrm{F}, \sigma}^{p}(k)\right], \\
k_{\mathrm{F}, \sigma}^{p}(k) & =k_{\mathrm{F}}^{0}+\sigma \frac{h}{2 v}+\frac{2 t_{1}}{v} \cos k-p \frac{2 t_{2}}{v} \sin k,
\end{aligned}
$$

where $p=+/-$ (or R/L), $k_{\mathrm{F}}^{0}=\pi / 4, v=\sqrt{2} t_{\|}, \sigma=+/-(=\uparrow$ $/ \downarrow), t_{1} \equiv t_{\perp 1}+t_{\perp 2} / \sqrt{2}$, and $t_{2} \equiv t_{\perp 2} / \sqrt{2}$. Terms of $O\left(t_{\perp}^{2}\right)$ and $k$ dependence of the Fermi velocity are discarded.
Expressing the interaction in terms of forward and backward scattering, ${ }^{17)}$ eq. (3) is rewritten as

$$
\begin{aligned}
& H_{\mathrm{I}}=\frac{2 \pi v}{L N_{\perp}} \sum_{\boldsymbol{k}_{1}, \boldsymbol{k}_{2}, \boldsymbol{q},\{\sigma\}} G_{\{\sigma\}\left(q, k_{1}, k_{2}\right)} \\
& \times c_{R, \sigma_{1}}^{\dagger}\left(\boldsymbol{k}_{\mathbf{1}}\right) c_{L, \sigma_{2}}\left(\boldsymbol{k}_{\mathbf{1}}-\boldsymbol{q}\right) c_{L, \sigma_{3}}^{\dagger}\left(\boldsymbol{k}_{\mathbf{2}}-\boldsymbol{q}\right) c_{R, \sigma_{4}}\left(\boldsymbol{k}_{\mathbf{2}}\right),
\end{aligned}
$$

where

$$
\begin{aligned}
G_{\{\sigma\}\left(q, k_{1}, k_{2}\right)} & =G_{1 \perp\left(q, k_{1}, k_{2}\right)} \delta_{\sigma_{1}, \sigma_{2}} \delta_{\sigma_{3}, \sigma_{4}} \delta_{\sigma_{1},-\sigma_{3}} \\
& -G_{2 \perp\left(q, k_{1}, k_{2}\right)} \delta_{\sigma_{1}, \sigma_{4}} \delta_{\sigma_{2}, \sigma_{3}} \delta_{\sigma_{1},-\sigma_{3}} \\
& -G_{\|\left(q, k_{1}, k_{2}\right)} \delta_{\sigma_{1}, \sigma_{4}} \delta_{\sigma_{2}, \sigma_{3}} \delta_{\sigma_{1}, \sigma_{3}} .
\end{aligned}
$$

The couplings $G_{2} \perp$ and $G_{\|}$stand for the forward scattering with spin being anti-parallel and parallel, respectively. The amplitude $G_{1 \perp}$ denotes that of the backward scattering with anti-parallel spins. The $8 k_{\mathrm{F}}-$ Umklapp scattering due to the quarter-filling is discarded. The coupling constants depend on the wave vector perpendicular to the chains, ${ }^{21)}$ and the definition is the same as in ref. 24. The bare scattering amplitudes, which correspond to the initial values for the RG equations, are given by

$$
\begin{aligned}
G_{1 \perp\left(q, k_{1}, k_{2}\right)} & =\frac{1}{2 \pi v}\left(U+2 V_{\perp}^{\mathrm{b}} \cos q\right), \\
G_{2 \perp\left(q, k_{1}, k_{2}\right)} & =\frac{1}{2 \pi v}\left[U+2 V_{\perp}^{\mathrm{f}} \cos \left(k_{1}-k_{2}\right)\right], \\
G_{\|\left(q, k_{1}, k_{2}\right)} & =\frac{1}{2 \pi v}\left[2 V_{\perp}^{\mathrm{f}} \cos \left(k_{1}-k_{2}\right)-2 V_{\perp}^{\mathrm{b}} \cos q\right] .
\end{aligned}
$$

In the following, we shall only retain the backscattering part $\left(V_{\perp}^{\mathrm{b}}\right)$ and neglect the forward scattering contribution $V_{\perp}^{\mathrm{f}}$ of eqs. (9a)-(9c); $V_{\perp}^{\mathrm{b}}$ is known to be involved in the enhancement of $2 k_{\mathrm{F}}^{0} \mathrm{CDW}$ fluctuations. ${ }^{23,25)}$

\subsection{RG equations for the vertex couplings}

We consider the partition function, which is represented in the path integral form,

$$
Z=\iint \mathscr{D} \psi^{*} \mathscr{D} \psi e^{S\left[\psi^{*}, \psi\right]}
$$

where $S\left[\psi^{*}, \psi\right]=S_{0}\left[\psi^{*}, \psi\right]+S_{\mathrm{I}}\left[\psi^{*}, \psi\right]$ is the action corresponding to the Hamiltonian (1). The fields $\psi^{(*)}$ are the Grassmann variables for the electron degrees of freedom and $D \psi^{*} \mathscr{D} \psi$ corresponds to the integration measure for the Grassmann variables. In the Fourier-Matsubara space, the free and interacting parts of the action $S_{0}\left[\psi^{*}, \psi\right]$ and $S_{\mathrm{I}}\left[\psi^{*}, \psi\right]$ are respectively given by ${ }^{18)}$

$$
\begin{aligned}
& S_{0}\left[\psi^{*}, \psi\right]=\sum_{\boldsymbol{k}, i \omega_{n}, \sigma, p}\left[g_{p, \sigma}^{0}\left(\boldsymbol{k}, i \omega_{n}\right)\right]^{-1} \psi_{p, \sigma}^{*}(\tilde{\boldsymbol{k}}) \psi_{p, \sigma}(\tilde{\boldsymbol{k}}), \\
& S_{\mathrm{I}}\left[\psi^{*}, \psi\right]=-T \sum_{i \omega_{n_{1}}, i \omega_{n_{2}}, i \omega_{m}} H_{\mathrm{I}}^{\mathrm{G}}\left[\psi^{*}, \psi\right],
\end{aligned}
$$

where $T$ is the temperature ( $k_{B}=1$ throughout this work) and $H_{\mathrm{I}}^{\mathrm{G}}\left[\psi^{*}, \psi\right]$ is obtained by substituting the fermion operators for the Grassmann variable e.g., $c_{p, \sigma}(\boldsymbol{k}-\boldsymbol{q}) \rightarrow \psi_{p, \sigma}(\tilde{\boldsymbol{k}}-\tilde{\boldsymbol{q}})$, etc., where $\tilde{\boldsymbol{k}}=\left(k_{\|}, k, \omega_{n}\right)$ and $\tilde{\boldsymbol{q}}=\left(q_{\|}, q, \omega_{m}\right)$, with $\omega_{n}$ and $\omega_{m}$ being the Matsubara frequencies for fermions and bosons, respectively. The Green's function for the free fermions is given by $g_{p, \sigma}^{0}\left(\boldsymbol{k}, i \omega_{n}\right)=\left[i \omega_{n}-\varepsilon_{p, \sigma}(\boldsymbol{k})\right]^{-1}$. In order to examine the behavior at low temperature, we proceed to implement 
(a)

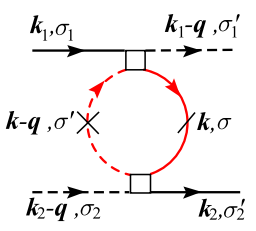

(b)

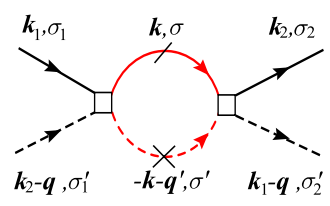

Fig. 2. (Color online) Peierls (a) and Cooper (b) bubbles. Solid (broken) line represent right (left) going electron, $p=+(-)$; the square is the interaction vertex i.e., $G_{1 \perp}, G_{2 \perp}$, and $G_{\|}$. Slashed lines represent electrons on high-energy shell $E_{l+d l}<\left|\varepsilon_{p, \sigma}(\boldsymbol{k})\right|<E_{l}$, while crossed lines represent electrons at higher energy or lower $l$ values.

the successive partial integrations of eq. (10) on high-energy shells. This leads to the renormalization of the inner shell action $\left(S_{<}\right)$at step $l$ of the procedure, where $l \geq 0$ is a RG parameter for the scaled energy $E_{l}=E e^{-l}\left(2 E_{l}\right.$ is the scaled bandwidth at $l$ ). ${ }^{21)}$ The partial integration of high-energy outer shell degrees of freedom is performed perturbatively with respect to $S_{0}$. The use of the linked cluster expansion allows one to write the result in the form

$$
Z \propto \iint_{<} \mathscr{D} \psi^{*} \mathscr{D} \psi \exp \left[S_{<}\left[\psi^{*}, \psi\right]+\sum_{n=1}^{\infty} \frac{1}{n !}\left\langle S_{\mathrm{I}>}^{n}\right\rangle_{0, \mathrm{c}}\right],
$$

where $\langle\cdots\rangle_{0, \mathrm{c}}$ denotes the contribution of connected diagrams to the outer energy shell of width $E_{l} d l$, where $d l \ll 1$. The action $S_{l+d l}$, at the step $l+d l$, thus contains additional renormalization terms, which for the coupling constants read

$$
\begin{aligned}
\delta G_{v\left(q, k_{1}, k_{2}\right)}(l) & =G_{v\left(q, k_{1}, k_{2}\right)}(l+d l)-G_{v\left(q, k_{1}, k_{2}\right)}(l) \\
& =\delta G_{v\left(q, k_{1}, k_{2}\right)}^{n=2}+\delta G_{v\left(q, k_{1}, k_{2}\right)}^{n=3}+\cdots,
\end{aligned}
$$

where $v=1 \perp, 2 \perp$, and $\|$. The logarithmic contributions at the one-loop level $(n=2)$ for $\delta G_{v\left(q, k_{1}, k_{2}\right)}^{n=2}$ are given by the diagrams shown in Fig. 2.

This renormalization consists of two parts, which come from the Peierls channel and Cooper channels, namely

$$
\delta G_{v\left(q, k_{1}, k_{2}\right)}^{n=2}=F_{\mathrm{P}\left(q, k_{1}, k_{2}\right)}^{v}+F_{\mathrm{C}\left(q, k_{1}, k_{2}\right)}^{v} .
$$

The Peierls bubble $F_{\mathrm{P}}^{v}\left(q, k_{1}, k_{2}\right)$ is given by

$$
\begin{aligned}
& F_{\mathrm{P}\left(q, k_{1}, k_{2}\right)}^{v}=-\frac{2 \pi v T}{L N_{\perp}} \sum_{v_{1}, v_{2}} \sum_{\boldsymbol{k}}^{\text {o.s.' }} \sum_{i \omega_{n}} \\
& \quad \times J_{\mathrm{P}\left(q, v_{1}, v_{2}, k_{1}, k_{2}\right)}^{v, g_{R, \sigma}}\left(\boldsymbol{k}, i \omega_{n}\right) g_{L, \sigma^{\prime}}^{0}\left(\boldsymbol{k}-\boldsymbol{q}, i \omega_{n}\right),
\end{aligned}
$$

where

$$
J_{\mathrm{P}\left(q, k, k_{1}, k_{2}\right)}^{v, v_{1}, v_{2}}=(-1)^{m_{p}^{v, v_{1}, v_{2}}} G_{v_{1}\left(q, k_{1}, k\right)} G_{v_{2}\left(q, k, k_{2}\right)},
$$

and the summation of $v_{1}, v_{2}(=1 \perp, 2 \perp, \|)$ is taken for the fixed $v$, as shown explicitly later. The quantity $m_{p}^{v, v_{1}, v_{2}}$ denotes the number of the permutation of Grassmann variable. $\sum_{k}^{\text {o.s.' }}$ is performed in the outer shell region. The summation is written as

$$
\sum_{\boldsymbol{k}}^{o . s . .^{\prime}}=2 \sum_{\boldsymbol{k}}^{o . s .} \Theta\left(\left|\varepsilon_{L, \sigma^{\prime}}(\boldsymbol{k}-\boldsymbol{q})\right|-E_{l}\right),
$$

where $\sum_{k}^{o . s .}$ denotes the summation in the region of $E_{l+d l}<$ $\left|\varepsilon_{R, \sigma}(\boldsymbol{k})\right|<E_{l}$ and $\Theta(x)$ is the Heaviside step function with the definition $\Theta(0) \equiv 1 / 2$. In the RG procedure, the external momentum is fixed at the Fermi sur- face, i.e., $\left(k_{\mathrm{F}, \sigma_{1}}^{R}\left(k_{1}\right), k_{1}\right),\left(-k_{\mathrm{F}, \sigma_{2}^{\prime}}^{L}\left(k_{2}-q\right), k_{2}-q\right)$ for the incoming states and $\left(k_{\mathrm{F}, \sigma_{2}}^{R}\left(k_{2}\right), k_{2}\right),\left(-k_{\mathrm{F}, \sigma_{1}^{\prime}}^{L}\left(k_{1}-q\right), k_{1}-q\right)$ for the outgoing states. Thus for the momentum summation of the bubble with $\boldsymbol{q}=\left(q_{\|}, q\right)$, the longitudinal momentum $q_{\|}$is determined by the momentum conservation for the respective vertex $\left(v_{1}, v_{2}\right){ }^{24)}$ Using $\left(L N_{\perp}\right)^{-1} \sum_{k}^{\text {o.s. }}=$ $\left(2 \pi v N_{\perp}\right)^{-1} \sum_{k} \int_{\text {o.s. }} d \varepsilon_{R, \sigma}(\boldsymbol{k})$, and performing the Matsubarafrequency summation, eq. (16) is rewritten as

$$
\begin{aligned}
& F_{\mathrm{P}\left(q, k_{1}, k_{2}\right)}^{v}=-\frac{2}{N_{\perp}} \sum_{v_{1}, v_{2}} \sum_{k} J_{\mathrm{P}\left(q, k, k_{1}, k_{2}\right)}^{v, v_{1}, v_{2}} \\
& \quad \times \int_{\text {o.s. }} d \varepsilon_{R, \sigma}(\boldsymbol{k}) \frac{f\left(\varepsilon_{R, \sigma}(\boldsymbol{k})\right)-f\left(\varepsilon_{L, \sigma^{\prime}}(\boldsymbol{k}-\boldsymbol{q})\right)}{\varepsilon_{R, \sigma}(\boldsymbol{k})-\varepsilon_{L, \sigma^{\prime}}(\boldsymbol{k}-\boldsymbol{q})} \\
& \quad \times \Theta\left(\left|\varepsilon_{L, \sigma^{\prime}}(\boldsymbol{k}-\boldsymbol{q})\right|-E_{l}\right),
\end{aligned}
$$

where $\int_{\text {o.s. }} d \varepsilon=\int_{E_{l+d l}}^{E_{l}} d \varepsilon+\int_{-E_{l}}^{-E_{l+d l}} d \varepsilon$. The Fermi distribution function $f(x)$ in eq. (19) is given by $f(x)=1 /[\exp (x / T)+1]$, which will be treated in the low-temperature limit. Following the $\mathrm{RG}$ procedure one can rescale the energy $2 E_{l}$ up to the original band width $2 E$, so that all the energies $A\left(\equiv t_{1}, t_{2}, h\right)$ are rescaled according to

$$
A(l)=A \mathrm{e}^{l},
$$

where the anomalous corrections to $A$ due to the two-loop corrections are small and have been neglected for the present choice of parameters (see Appendix). At zero temperature, eq. (19) is calculated as

$$
\begin{aligned}
F_{\mathrm{P}\left(q, k_{1}, k_{2}\right)}^{v}= & \frac{1}{N_{\perp}} \sum_{v_{1}, v_{2}} \sum_{k} J_{\mathrm{P}\left(q, k, k_{1}, k_{2}\right)}^{v, v_{1}, v_{2}} \\
& \times \frac{1}{2} \sum_{i=1,2} I_{\mathrm{P}\left(q, k, k_{i}, \sigma-\sigma_{i}\right)} d l
\end{aligned}
$$

where

$$
I_{\mathrm{P}\left(q, k, k_{i}, \sigma-\sigma_{i}\right)}=\frac{2 E}{2 E+\left|Y_{q, k, k_{i}, \sigma-\sigma_{i}}^{\mathrm{P}}(l)\right|}
$$

and

$$
\begin{aligned}
Y_{q, k, k_{i}, \sigma-\sigma_{i}}^{\mathrm{P}}(l) \equiv & -\varepsilon_{R, \sigma}(\boldsymbol{k})-\varepsilon_{L, \sigma^{\prime}}(\boldsymbol{k}-\boldsymbol{q}) \\
= & +2 t_{1}(l)[\cos k+\cos (k-q)] \\
& -2 t_{1}(l)\left[\cos k_{i}+\cos \left(k_{i}-q\right)\right] \\
& -2 t_{2}(l)[\sin k-\sin (k-q)] \\
& +2 t_{2}(l)\left[\sin k_{i}+\sin \left(k_{i}-q\right)\right] \\
& +h(l)\left(\sigma-\sigma_{i}\right) .
\end{aligned}
$$

In the above equations, $\sigma=\uparrow$ and $\downarrow$ are replaced by $\sigma=+1$ and -1 , respectively. In a similar way, the Cooper bubble is calculated as follows:

$$
\begin{aligned}
& F_{\mathrm{C}\left(q, k_{1}, k_{2}\right)}^{v}=-\frac{2 \pi v T}{L N_{\perp}} \sum_{v_{1}, v_{2}} \sum_{k}^{\text {o.s. }} \sum_{i \omega_{n}}^{\prime \prime} \\
& \quad \times J_{\mathrm{C}\left(q, k, k_{1}, k_{2}\right)}^{v, v_{1}, v_{2}} g_{R, \sigma}^{0}\left(\boldsymbol{k}, i \omega_{n}\right) g_{L, \sigma^{\prime}}^{0}\left(-\boldsymbol{k}-\boldsymbol{q}^{\prime},-i \omega_{n}\right),
\end{aligned}
$$

where $\boldsymbol{q}^{\prime} \equiv \boldsymbol{q}-\boldsymbol{k}_{\mathbf{1}}-\boldsymbol{k}_{\mathbf{2}}$ and

$$
J_{\mathrm{C}\left(q, k, k_{1}, k_{2}\right)}^{v, v_{1}, v_{2}}=(-1)^{m_{p}^{v, v_{1}, v_{2}}} G_{v_{1}\left(q-k_{2}+k, k_{1}, k\right)} G_{v_{2}\left(q-k_{1}+k, k, k_{2}\right)},
$$




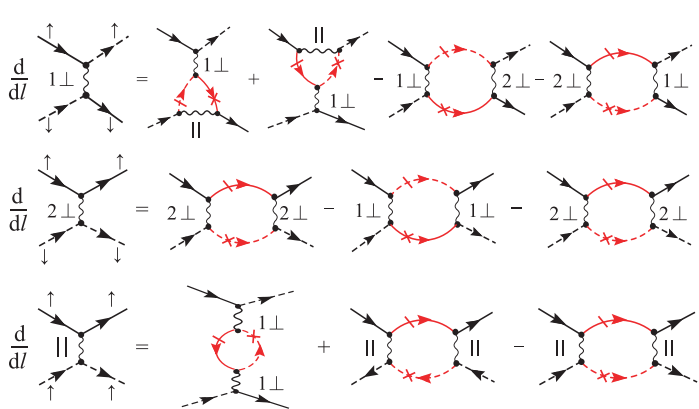

Fig. 3. (Color online) The RG equations for the coupling constants at the one-loop level. The slashed and crossed internal lines represent the electrons on the high-energy shell and within the shell, respectively. The diagrams with the slash and cross lines interchanged (not shown) are also taken into account.

and the summation $\sum_{k}^{\text {o.s. }}$ is given by substituting $\varepsilon_{L, \sigma^{\prime}}(-\boldsymbol{k}-$ $\left.\boldsymbol{q}^{\prime}\right)$ for $\varepsilon_{L, \sigma^{\prime}}(\boldsymbol{k}-\boldsymbol{q})$ in eq. (18). The Fermi surface of the external momentum is given by $\left(k_{\mathrm{F}, \sigma_{1}}^{R}\left(k_{1}\right), k_{1}\right),\left(-k_{\mathrm{F}, \sigma_{1}^{\prime}}^{L}\left(k_{2}-\right.\right.$ $\left.q), k_{2}-q\right)$ for the incoming states, and $\left(k_{\mathrm{F}, \sigma_{2}}^{R}\left(k_{2}\right), k_{2}\right)$, $\left(-k_{\mathrm{F}, \sigma_{2}^{\prime}}^{L}\left(k_{1}-q\right), k_{1}-q\right)$ for the outgoing states. The longitudinal momentum vector $q_{\|}^{\prime}$ is also determined by the momentum conservation for respective vertex. Performing the Matsubarafrequency summation, eq. (24) is rewritten as

$$
\begin{aligned}
& F_{\mathrm{C}\left(q, k_{1}, k_{2}\right)}^{v}=\frac{2}{N_{\perp}} \sum_{k} \sum_{v_{1}, v_{2}} J_{\mathrm{C}\left(q, k, k_{1}, k_{2}\right)}^{v, v_{1}, v_{2}} \\
& \quad \times \int_{\text {o.s. }} d \varepsilon_{R, \sigma}(\boldsymbol{k}) \frac{f\left(\varepsilon_{R, \sigma}(\boldsymbol{k})\right)-f\left(-\varepsilon_{L, \sigma^{\prime}}\left(-\boldsymbol{k}-\boldsymbol{q}^{\prime}\right)\right)}{\varepsilon_{R, \sigma}(\boldsymbol{k})+\varepsilon_{L, \sigma^{\prime}}\left(-\boldsymbol{k}-\boldsymbol{q}^{\prime}\right)} \\
& \quad \times \Theta\left(\left|\varepsilon_{L, \sigma^{\prime}}\left(-\boldsymbol{k}-\boldsymbol{q}^{\prime}\right)\right|-E_{l}\right) .
\end{aligned}
$$

At zero temperature, eq. (26) is calculated as

$$
\begin{aligned}
F_{\mathrm{C}\left(q, k_{1}, k_{2}\right)}^{v}= & -\frac{1}{N_{\perp}} \sum_{v_{1}, v_{2}} \sum_{k} J_{\mathrm{C}\left(q, k, k_{1}, k_{2}\right)}^{v, v_{1}, v_{2}} \\
& \times \frac{1}{2} \sum_{i=1,2} I_{\mathrm{C}\left(q^{\prime}, k, k_{i}, \sigma-\sigma_{i}\right)},
\end{aligned}
$$

where

$$
I_{\mathrm{C}\left(q^{\prime}, k, k_{i}, \sigma-\sigma_{i}\right)}=\frac{2 E}{2 E+\left|Y_{q^{\prime}, k, k_{i}, \sigma-\sigma_{i}}^{\mathrm{C}}(l)\right|},
$$

and

$$
\begin{aligned}
Y_{q^{\prime}, k, k_{i}, \sigma-\sigma_{i}}^{\mathrm{C}}(l) \equiv & \varepsilon_{R, \sigma}(\boldsymbol{k})+\varepsilon_{L, \sigma^{\prime}}\left(-\boldsymbol{k}-\boldsymbol{q}^{\prime}\right) \\
= & +2 t_{1}(l)\left[\cos k-\cos \left(k+q^{\prime}\right)\right] \\
& -2 t_{1}(l)\left[\cos k_{i}-\cos \left(k_{i}+q^{\prime}\right)\right] \\
& -2 t_{2}(l)\left[\sin k-\sin \left(k+q^{\prime}\right)\right] \\
& +2 t_{2}(l)\left[\sin k_{i}-\sin \left(k_{i}+q^{\prime}\right)\right] \\
& +h(l)\left(\sigma-\sigma_{i}\right) .
\end{aligned}
$$

The functions $I_{\mathrm{P}\left(q, k, k_{i}, \sigma-\sigma_{i}\right)}$ and $I_{\mathrm{C}\left(q, k, k_{i}, \sigma-\sigma_{i}\right)}$, which are equal to unity in the one-dimensional case and in the absence of the magnetic field, are reduced by $t_{1}$ and $t_{2}$ in the quasi-1D case.

The RG flow equations at the one-loop level are shown diagrammatically in Fig. 3. Including the two-loop corrections derived in the Appendix, the RG equations take the form

$$
\begin{aligned}
\frac{d}{d l} G_{v\left(q, k_{1}, k_{2}\right)} & =\frac{1}{2 N_{\perp}} \sum_{k} \Xi_{v\left(q, k, k_{1}, k_{2}\right)}^{(1)} \\
& -\frac{1}{8 N_{\perp}^{2}} G_{v}\left(q, k_{1}, k_{2}\right) \sum_{q^{\prime}, k^{\prime}} \Xi_{\Sigma\left(q, k_{1}, k_{2}, q^{\prime}, k^{\prime}\right)}^{(2)} \\
& +\frac{1}{4 N_{\perp}^{2}} \sum_{q^{\prime}, k^{\prime}} \Xi_{v\left(q, k_{1}, k_{2}, q^{\prime}, k^{\prime}\right)}^{(2)}+\left(k_{1} \leftrightarrow k_{2}\right),
\end{aligned}
$$

where $\Xi_{v\left(q, k, k_{1}, k_{2}\right)}^{(1)}$ is the contribution from the one-loop RG, while $\Xi_{\Sigma\left(q, k_{1}, k_{2}, q^{\prime}, k^{\prime}\right)}^{(2)}$ and $\Xi_{v\left(q, k_{1}, k_{2}, q^{\prime}, k^{\prime}\right)}^{(2)}$ are the two-loop contributions coming from the self-energy and the vertex part, respectively (see Appendix). ${ }^{24)}$ The quantity $\Xi_{v\left(q, k, k_{1}, k_{2}\right)}^{(1)}$ is written as

$$
\begin{aligned}
& \Xi_{1 \perp\left(q, k, k_{1}, k_{2}\right)}^{(1)}=G_{1 \perp\left(q, k_{1}, k\right)} G_{\|\left(q, k, k_{2}\right)} I_{\mathrm{P}\left(q, k, k_{1}, k_{2}\right)}^{(1)} \\
& \quad+G_{\|\left(q, k_{1}, k\right)} G_{1 \perp\left(q, k, k_{2}\right)} I_{\mathrm{P}\left(q, k, k_{2}, k_{1}\right)}^{(1)} \\
& \quad-G_{1 \perp\left(q-k_{2}+k, k_{1}, k\right)} G_{2 \perp\left(q-k_{1}+k, k, k_{2}\right)} I_{\mathrm{C}\left(q-k_{1}-k_{2}, k, k_{1}, k_{2}\right)}^{(1)} \\
& \quad-G_{2 \perp\left(q-k_{2}+k, k_{1}, k\right)} G_{1 \perp\left(q-k_{1}+k, k, k_{2}\right)} I_{\mathrm{C}\left(q-k_{1}-k_{2}, k, k_{2}, k_{1}\right)}^{(1)},
\end{aligned}
$$

$$
\begin{aligned}
& \Xi_{2 \perp\left(q, k, k_{1}, k_{2}\right)}^{(1)}=G_{2 \perp\left(q, k_{1}, k\right)} G_{2 \perp\left(q, k, k_{2}\right)} I_{\mathrm{P}\left(q, k, k_{1}, k_{2}\right)}^{(0)} \\
& \quad-G_{1 \perp\left(q-k_{2}+k, k_{1}, k\right)} G_{1 \perp\left(q-k_{1}+k, k, k_{2}\right)} I_{\mathrm{C}\left(q-k_{1}-k_{2}, k, k_{1}, k_{2}\right)}^{(2)} \\
& \quad-G_{2 \perp\left(q-k_{2}+k, k_{1}, k\right)} G_{2 \perp\left(q-k_{1}+k, k, k_{2}\right)} I_{\mathrm{C}\left(q-k_{1}-k_{2}, k, k_{1}, k_{2}\right)}^{(0)},
\end{aligned}
$$

$$
\begin{aligned}
& \Xi_{\|\left(q, k, k_{1}, k_{2}\right)}^{(1)}=G_{1 \perp\left(q, k_{1}, k\right)} G_{1 \perp\left(q, k, k_{2}\right)} I_{\mathrm{P}\left(q, k, k_{1}, k_{2}\right)}^{(2)} \\
& \quad+G_{\|\left(q, k_{1}, k\right)} G_{\|\left(q, k, k_{2}\right)} I_{\mathrm{P}\left(q, k, k_{1}, k_{2}\right)}^{(0)} \\
& \quad-G_{\|\left(q-k_{2}+k, k_{1}, k\right)} G_{\|\left(q-k_{1}+k, k, k_{2}\right)} I_{\mathrm{C}\left(q-k_{1}-k_{2}, k, k_{1}, k_{2}\right)}^{(0)}
\end{aligned}
$$

where for $\lambda=\mathrm{P}, \mathrm{C}$,

$$
\begin{aligned}
I_{\lambda\left(q, k, k_{1}, k_{2}\right)}^{(0)} & =\frac{1}{2} \sum_{i=1,2} I_{\lambda\left(q, k, k_{i}, 0\right)}, \\
I_{\lambda\left(q, k, k_{1}, k_{2}\right)}^{(1)} & =\frac{1}{4} \sum_{r= \pm 1}\left[I_{\lambda\left(q, k, k_{1}, 2 r\right)}+I_{\lambda\left(q, k, k_{2}, 0\right)}\right] \\
I_{\lambda\left(q, k, k_{1}, k_{2}\right)}^{(2)} & =\frac{1}{4} \sum_{r= \pm 1} \sum_{i=1,2} I_{\lambda\left(q, k, k_{i}, 2 r\right)} .
\end{aligned}
$$

Note that in the absence of interchain couplings, $\Xi_{v\left(q, k, k_{1}, k_{2}\right)}^{(1)}$ coincides with the expressions already obtained by Montambaux et al., ${ }^{20)}$ at $h \neq 0$ in the 1D case.

\subsection{RG equations for response functions}

Now we calculate the response functions for CDW, SDW, $\mathrm{SC} d$, and $\mathrm{SC} f$. The composite fields of corresponding order parameters are defined as

$\mathscr{O}_{\mathrm{CDW}}\left(\boldsymbol{q}_{\mathrm{P}}\right)=\sqrt{\frac{1}{L N_{\perp}}} \sum_{\boldsymbol{k}, \sigma} c_{R, \sigma}^{\dagger}(\boldsymbol{k}) c_{L, \sigma}\left(\boldsymbol{k}-\boldsymbol{q}_{\mathrm{P}}\right)$, 
(a)

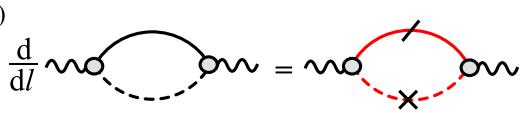

(b)

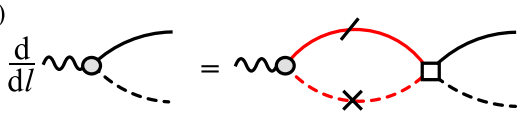

Fig. 4. (Color online) Renormalization group equations for the response functions (a), and order parameter vertex part for the source field (b).

$$
\begin{aligned}
& \mathscr{O}_{\mathrm{SDW}}\left(\boldsymbol{q}_{\mathrm{P}}\right)=\sqrt{\frac{1}{L N_{\perp}}} \sum_{\boldsymbol{k}, \sigma} c_{R, \sigma}^{\dagger}(\boldsymbol{k}) c_{L,-\sigma}\left(\boldsymbol{k}-\boldsymbol{q}_{\mathrm{P}}\right), \\
& \mathscr{O}_{\mathrm{SC} d}\left(\boldsymbol{q}_{\mathrm{C}}\right)=\sqrt{\frac{1}{L N_{\perp}}} \sum_{\boldsymbol{k}, \sigma}(\sigma \cos k) c_{R, \sigma}(\boldsymbol{k}) c_{L,-\sigma}\left(-\boldsymbol{k}+\boldsymbol{q}_{\mathrm{C}}\right), \\
& \mathscr{O}_{\mathrm{SC} f}\left(\boldsymbol{q}_{\mathrm{C}}\right)=\sqrt{\frac{1}{L N_{\perp}}} \sum_{\boldsymbol{k}, \sigma}\left(\sin k_{\|} \cos k\right) c_{R, \sigma}(\boldsymbol{k}) c_{L, \sigma}\left(-\boldsymbol{k}+\boldsymbol{q}_{\mathrm{C}}\right),
\end{aligned}
$$

where the density-wave modulation $\boldsymbol{q}_{\mathrm{P}}=\left(2 k_{\mathrm{F}}^{0}, \pi\right)$ is the nesting vector and $\boldsymbol{q}_{\mathrm{C}}=(0,0)$.

For simplicity, we use the four patches model for the Fermi surface, for which $k=0, \pi$, as shown in Fig. 1 (b). We thus end up with 12 independent coupling constants. Such a choice allows a qualitative description of the SC states, i.e., eqs. (33c) and (33d) having respectively the gap functions $\cos k$ and $\sin k_{\|} \cos k$. This corresponds to nodes located at $k= \pm \pi / 2$ for $\mathrm{SC} d$, and $k_{\|}=0$ and $k= \pm \pi / 2$ for SC $f$. Hereafter, we use 0 or $\pi$ for the momentum perpendicular to the chain. While the $t_{2}$-terms in eqs. (23) and (29) vanish for $q, k, k_{i}=0, \pi$, nesting deviations can be incorporated in the Peierls channel by introducing the following factor for the Peierls channel in eq. (30),

$$
I_{t_{2}^{*}}=\frac{E}{E+t_{2}^{*}(l)},
$$

where $t_{2}^{*}\left(\propto t_{2}\right)$ is a cut-off energy. It follows that $I_{t_{2}^{*}} \simeq 1$ for $E \gg t_{2}^{*}(l)$ and $I_{t_{2}^{*}} \simeq 0$ for $E \ll t_{2}^{*}(l)$. A cutoff procedure similar to eq. (34) has been used in studying the density-wave problem in many-coupled-chains case. ${ }^{28)}$

We calculate the response functions by adding a linear coupling of order parameters to source fields in the action, ${ }^{18)}$ that is $S_{h}\left[\psi, \psi^{*}, h, h^{*}\right]=\sum_{\mu}\left(h_{\mu} \mathscr{O}_{\mu}^{*}+\right.$ c.c. $)$, where $h_{\mu}$ is a source field in the $\mu=\mathrm{CDW}, \mathrm{SDW}, \mathrm{SC} d$, and SC $f$ channels. The total action at step $l$ is given by

$$
\begin{aligned}
& S\left[\psi, \psi^{*}, h, h^{*}\right]_{l}=S\left[\psi^{*}, \psi\right]_{l} \\
& +\sum_{\mu}\left(h_{\mu} z_{\mu}(l) \mathscr{O}_{\mu}^{*}+\text { c.c. }\right)+\chi_{\mu}(l) h_{\mu} h_{\mu}^{*},
\end{aligned}
$$

where $z_{\mu}$ is a renormalization factor for the order parameter vertex $\left(z_{\mu}(0)=1\right)$, and $\chi_{\mu}(l)$ is the response function with $\chi_{\mu}(0)=0$.

Up to the one-loop level (see Fig. 4), the RG equations for these quantities are

$$
\frac{d}{d l} \chi_{\mathrm{CDW}}\left(\boldsymbol{q}_{\mathrm{P}}\right)=\frac{1}{\pi v} z_{\mathrm{CDW}}^{2}\left(\boldsymbol{q}_{\mathrm{P}}\right) I_{h / 2} I_{t_{2}^{*}},
$$

$$
\begin{aligned}
\frac{d}{d l} \chi_{\mathrm{SDW}}\left(\boldsymbol{q}_{\mathrm{P}}\right) & =\frac{1}{\pi v} z_{\mathrm{SDW}}^{2}\left(\boldsymbol{q}_{\mathrm{P}}\right) I_{t_{2}^{*}}, \\
\frac{d}{d l} \chi_{\mathrm{SC} d}\left(\boldsymbol{q}_{\mathrm{C}}\right) & =\frac{1}{\pi v} z_{\mathrm{SC} d}^{2}\left(\boldsymbol{q}_{\mathrm{C}}\right) I_{h / 2}, \\
\frac{d}{d l} \chi_{\mathrm{SC} f}\left(\boldsymbol{q}_{\mathrm{C}}\right) & =\frac{1}{\pi v} z_{\mathrm{SC} f}^{2}\left(\boldsymbol{q}_{\mathrm{C}}\right),
\end{aligned}
$$

where the respective three-point vertices $z_{\mu}$ obey the RG equations:

$$
\begin{aligned}
\frac{d}{d l} \ln z_{\mathrm{CDW}}\left(\boldsymbol{q}_{\mathrm{P}}\right)= & {\left[-G_{1 \perp(\pi, 0,0)}-G_{1 \perp(\pi, 0, \pi)}\right.} \\
& \left.+G_{\|(\pi, 0, \pi)}+G_{\|(\pi, 0,0)}\right] I_{h / 2} I_{t_{2}^{*}}, \\
\frac{d}{d l} \ln z_{\mathrm{SDW}}\left(\boldsymbol{q}_{\mathrm{P}}\right)= & {\left[G_{2 \perp(\pi, 0, \pi)}+G_{2 \perp(\pi, 0,0)}\right] I_{t_{2}^{*}}, } \\
\frac{d}{d l} \ln z_{\mathrm{SC} d}\left(\boldsymbol{q}_{\mathrm{C}}\right)= & {\left[-G_{1 \perp(0,0,0)}+G_{1 \perp(\pi, 0, \pi)}\right.} \\
& \left.+G_{2 \perp(\pi, 0, \pi)}-G_{2 \perp(0,0,0)}\right] I_{h / 2}, \\
\frac{d}{d l} \ln z_{\mathrm{SC} f}\left(\boldsymbol{q}_{\mathrm{C}}\right)= & G_{\|(\pi, 0, \pi)}-G_{\|(0,0,0)} .
\end{aligned}
$$

The initial values are $\left.z_{\mu}\right|_{l=0}=1$. In the above equations, $I_{h}$ is defined by

$$
I_{h}=\frac{E}{E+h(l)} .
$$

Note that eq. (37b) represents the flow equation for the transverse SDW. The longitudinal SDW response is obtained by substituting $\left[G_{1 \perp(\pi, 0,0)}+G_{1 \perp(\pi, 0, \pi)}+G_{\|(\pi, 0, \pi)}+\right.$ $\left.G_{\|(\pi, 0,0)}\right] I_{h / 2} I_{t_{2}^{*}}$ for the r.h.s. of eq. (37b). These two flow equations become equivalent in the absence of $h$.

\section{Singlet versus triplet superconductivity}

For the numerical calculations that follow, parameters of the model are fixed at $U=4 t_{\|}$and $t_{1}=0.2 t_{\|}$unless stated explicitly. The unit of the energy is $t_{\|}$, which is set to unity $t_{\|}=1$, and we take $E=2 t_{\|}$. The scaling parameter $l$ is replaced by $l=\ln (E / T)$, where $T$ can be squared with the actual temperature introduced in $\S 2$.

In the following study of the four-patches Fermi surface, we will also examine the spin gap $\Delta_{\sigma}$, which is governed by the combination of coupling constants ${ }^{29)}$

$$
G_{\sigma+} \equiv \frac{1}{2}\left[G_{2 \perp(0,0,0)}-G_{\|(0,0,0)}+G_{2 \perp(\pi, 0,0)}-G_{\|(\pi, 0,0)}\right] .
$$

For a non zero $\Delta_{\sigma}$, the coupling $G_{\sigma+}$ takes a positive value at $l=0$, but moves to a fixed point with a large negative value. At a qualitative level for the spin gap, we shall use $\Delta_{\sigma}=E_{l_{\sigma}}\left(=E \mathrm{e}^{-l_{\sigma}}\right)$ where $l_{\sigma}$ is determined by the condition $G_{\sigma+}\left(l_{\sigma}\right)=-0.7 .^{24)}$ Thus a non zero spin gap is obtained for the couplings, $G_{2 \perp(0,0,0)}<0, G_{\|(0,0,0)}>0, G_{2 \perp(\pi, 0,0)}<0$, and $G_{\|(\pi, 0,0)}>0$. Here we note that in the absence of magnetic field, the combination of couplings for the spin gap, eq. (39), can be rewritten as

$$
G_{\sigma+}=\frac{1}{2}\left[G_{1 \perp(0,0,0)}+G_{1 \perp(\pi, 0,0)}\right] .
$$

From $G_{\sigma+}$, the uniform susceptibility within RPA takes the 

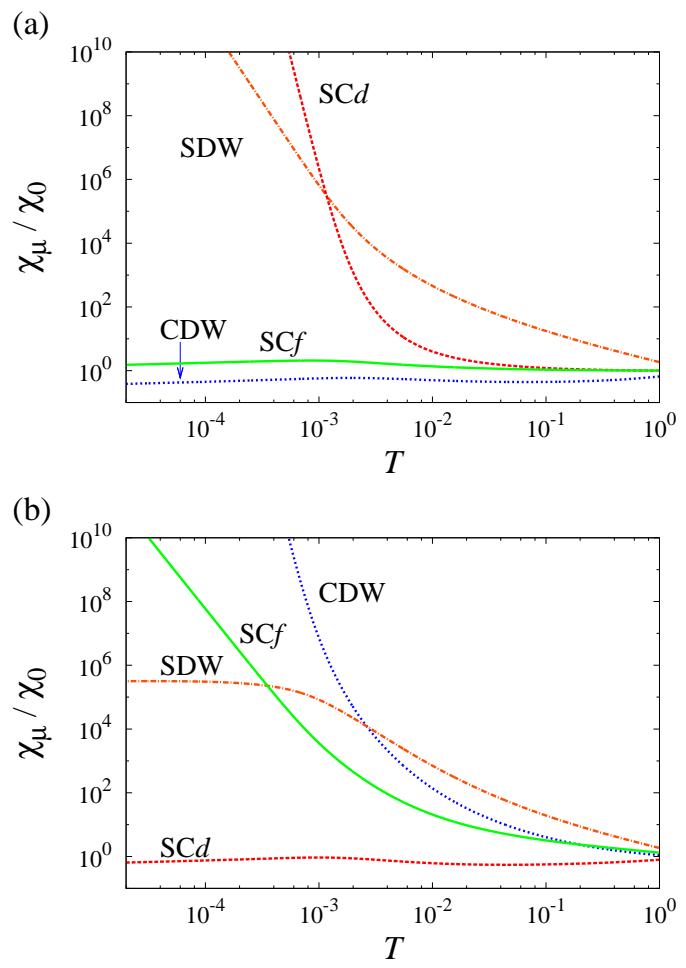

Fig. 5. (Color online) Temperature dependence of response function with $t_{2}^{*}=0.0, h=0.0$ for $V_{\perp}^{\mathrm{b}}=0.0$ (a), and 1.0 (b). Response functions $\chi_{\mu}$ are normalized by noninteracting response function $\chi_{0}(=l / \pi v)$.

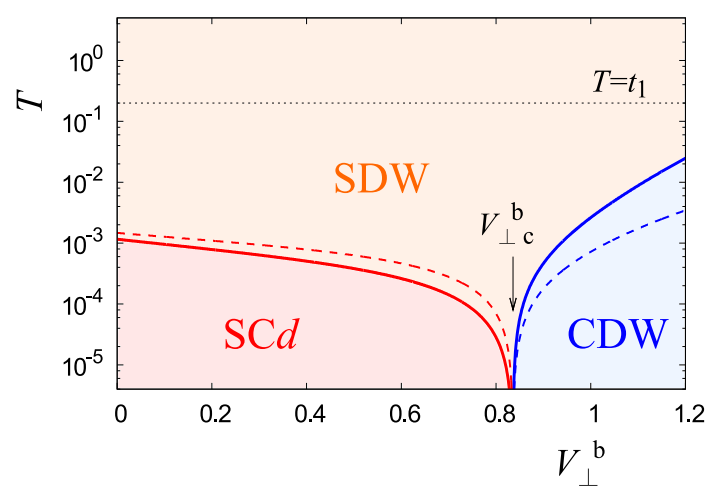

Fig. 6. (Color online) Possible states in the $V_{\perp}^{\mathrm{b}}-T$ plane with $t_{2}^{*}=0.0$ and $h=0.0$. The solid line represents the boundary of the respective phase, and the dotted line is the energy, $t_{1}$, corresponding to interchain hopping. The dashed line denotes the spin gap $\Delta_{\sigma}$ obtained from eq. (39).

form $^{30,31)}$

$$
\chi_{s}=\frac{\chi_{s 0}}{1-2 \pi \nu G_{\sigma+} \chi_{s 0}},
$$

where $\chi_{s 0}$ denotes the susceptibility for free electrons given by $2 \pi v \chi_{s 0}=\tanh (E / 2 T)$.

\subsection{Case of perfect nesting $\left(t_{2}^{*}=0\right)$}

Since the RG equations in the case of perfect nesting reduce to those obtained for two-coupled chains, ${ }^{29)}$ the dominant state in the absence of magnetic field is then either SCd or CDW. For $V_{\perp}^{\mathrm{b}}=0$, the system is characterized by a singlet SC $d$ state as shown in Fig. 5 (a). Such a result is well known for the Hubbard ladder model with repulsive interactions. ${ }^{32}$ ) The SDW correlations, which are the most dominant fluctua-

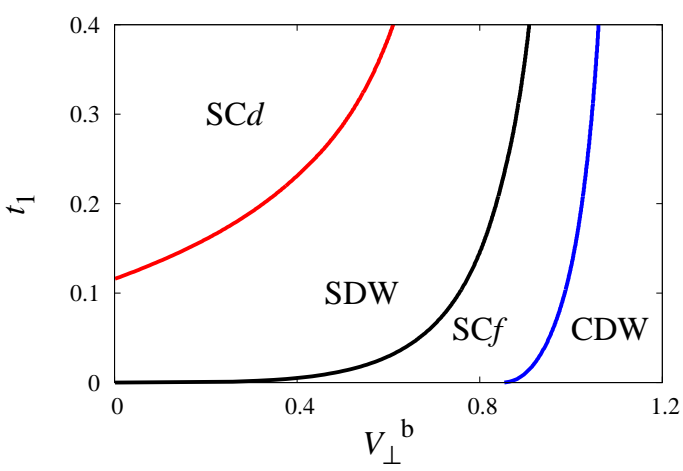

Fig. 7. (Color online) Phase diagram in the $V_{\perp}^{\mathrm{b}}-t_{1}$ plane with $h=0.0002$. The solid lines designate the boundary for respective phases. The line between $\mathrm{SDW}$ and $\mathrm{SC}_{f}$ refers to $V_{\perp \mathrm{c}}^{\mathrm{b}}$.

tions at high temperature, becomes less dominant at low temperature due to the formation of a spin gap. For $V_{\perp}^{\mathrm{b}}$ larger than a critical value, $V_{\perp c}^{\mathrm{b}}$, the CDW becomes in turn the dominant state as illustrated in Fig. 5 (b). The SC $f$ correlations are enhanced, but show a weaker increase than CDW at low temperature due to the spin gap.

In Fig. 6, the most dominant states are shown in the $V_{\perp}^{\mathrm{b}}-T$ plane. At high temperature $\left(T>t_{1}\right)$, corresponding to onedimensional regime, SDW is dominant. With decreasing temperature, the effect of interchain hoppings grows and the dominant state is in turn either SCd or CDW. On a temperature scale, this occurs at a characteristic temperature given by the solid line. This scale decreases and becomes zero at $V_{\perp}^{\mathrm{b}}=V_{\perp \mathrm{c}}^{\mathrm{b}}(\simeq 0.83)$; it increases monotonically for $V_{\perp}^{\mathrm{b}}>V_{\perp \mathrm{c}}^{\mathrm{b}}$. This behavior resembles to that of the spin gap, $\Delta_{\sigma}$, as shown by the dashed line in Fig. 6. The existence of such a critical point of $V_{\perp c}^{\mathrm{b}}$ has been also shown for two-coupled chains. ${ }^{29)}$ A similar behavior shown by $\Delta_{\sigma}$ indicates that spin degrees of freedom are also critical at $V_{\perp c}^{\mathrm{b}}$. The behavior around the quantum critical point, $V_{\perp c}^{\mathrm{b}}$, is ascribed to the competition between SCd and CDW, which are associated to different spin gaps. The spin gap for $\mathrm{SC} d$ is formed by interchain pairing, whereas that of CDW results from intrachain interactions. Therefore $\Delta_{\sigma}$ vanishes at the critical point where the symmetry of the gap changes.

We now turn to the effect of magnetic field, $h$, which brings new states due to its influence on the spin gap. When $\Delta_{\sigma}$ is destroyed by the magnetic field, the magnetic state is expected to be either the transverse SDW or SC $f$. Such a region actually exists when $V_{\perp}^{\mathrm{b}} \sim V_{\perp \mathrm{c}}^{\mathrm{b}}$. The phase diagram in the $V_{\perp}^{\mathrm{b}}-t_{1}$ plane is shown in Fig. 7. The boundary between SCd and SDW (and also between SC $f$ and $\mathrm{CDW}$ ) is estimated from the condition, $\Delta_{\sigma} \rightarrow 0$. It is found that the effect of $h$ on SCd is larger than it is for CDW. The SDW state thus takes place for $V_{\perp}^{\mathrm{b}}<V_{\perp \mathrm{c}}^{\mathrm{b}}$, whereas the $\mathrm{SC} f$ state appears for $V_{\perp}^{\mathrm{b}}>V_{\perp \mathrm{c}}^{\mathrm{b}}$. Moreover, $V_{\perp \mathrm{c}}^{\mathrm{b}}$ is a critical value for the crossover between SDW and SC $f$ as the dominant fluctuations. In our two-loop approach, the fact that both SDW and SC $f$ of Fig. 7 vanish for $h=0$ is at variance with the emergence of SDW state found in ref. 25 at low temperature using one-loop RG.

\subsection{Nesting deviations and superconductivity}

We now examine the SC state in the presence of nesting deviations, which is the main subject of the present paper. 
(a)

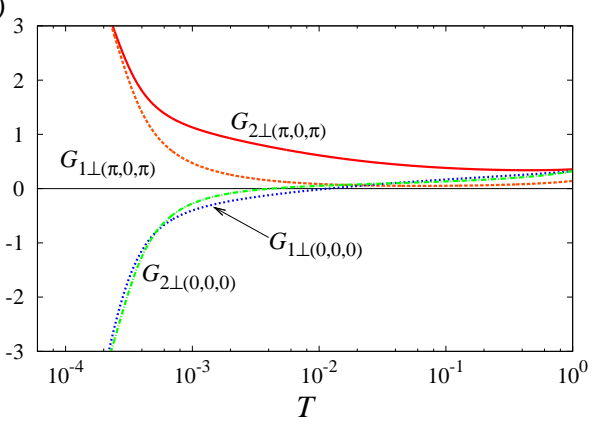

(b)

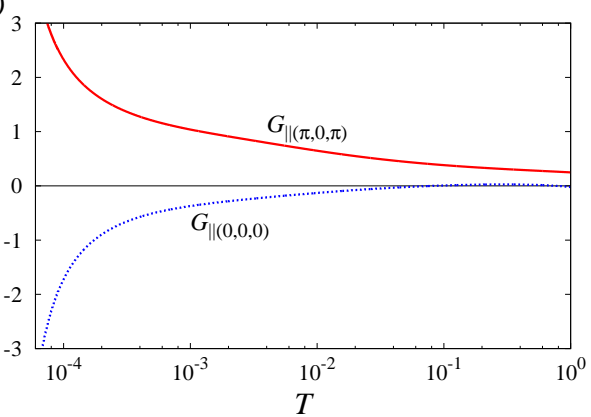

(c)

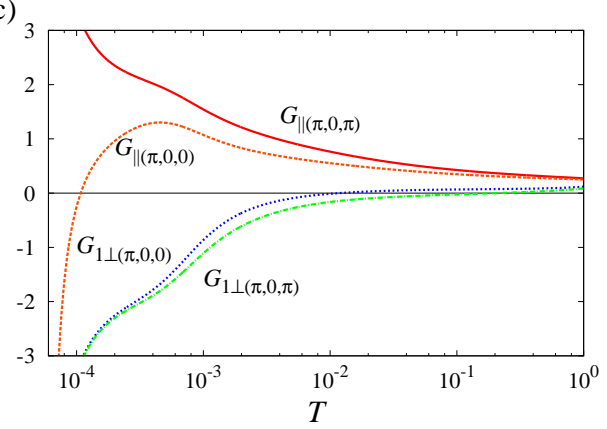

Fig. 8. (Color online) Temperature dependence of the coupling constants for $t_{2}^{*} / t_{1}=0.001$ and $h=0.0$, with fixed $V_{\perp}^{\mathrm{b}}=0.7$ (a), 0.88 (b), and 1.0 (c). Only the relevant coupling constants are shown.

We look at the possible states at $t_{2}^{*} \neq 0$ and low temperature by choosing $V_{\perp}^{\mathrm{b}}=0.7$ (a), 0.88 (b), and 1.0 (c), namely for small, intermediate, and large interchain couplings. Figures 8 (a), (b), and (c) show the temperature dependence of coupling constants which give rise to the response functions for $\mathrm{SC} d$, $\mathrm{SC} f$, and CDW respectively. The other couplings (not shown in the figures) only contribute a lesser degree to the response functions. These functions are traced in Figs. 9 (a)-(c).

For $V_{\perp}^{\mathrm{b}}=0.7$ [Fig. 8 (a)], the values of $G_{1 \perp(\pi, 0, \pi)}$ and $G_{2 \perp(\pi, 0, \pi)}$ at the fixed points are positive, while those of $G_{1 \perp(0,0,0)}$ and $G_{2 \perp(0,0,0)}$ are negative. The SC $d$ response function is then the strongest and becomes the dominant state. Since the coupling constant $G_{2 \perp(0,0,0)}$ changes its sign and becomes relevant, a spin gap appears [see eq. (39)], which is crucial to $\mathrm{SC} d$. As for the relevant coupling $G_{2 \perp(\pi, 0, \pi)}(>0)$, it enhances both SDW and $\mathrm{SC} d$, as seen from eqs. (37b) and (37c). Figure 9 (a) shows the temperature dependence of the response functions, where the SDW state is found to be the dominant state at high temperature, but becomes subdominant at low temperature. The amplitude of SDW correlations is reduced by nesting deviations. Thus it is found that $\mathrm{SC} d$ pairing is induced by spin fluctuations with the coupling $G_{2 \perp(\pi, 0, \pi)}(>0)$.
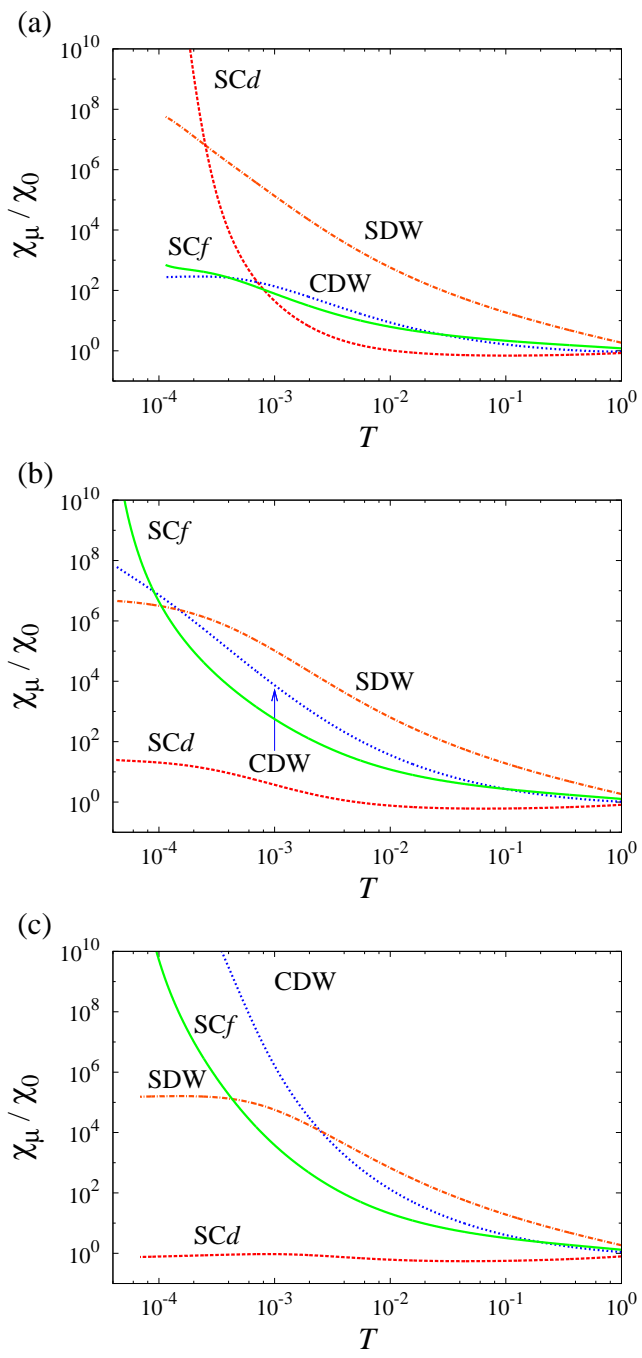

Fig. 9. (Color online) Temperature dependence of response functions at $t_{2}^{*} / t_{1}=0.001$ and $h=0.0$. with fixed $V_{\perp}^{\mathrm{b}}=0.7$ (a), $0.88(\mathrm{~b})$, and $1.0(\mathrm{c})$.

For $V_{\perp}^{\mathrm{b}}=0.88$ [Fig. 8 (b)], the relevant couplings are given by $G_{\|(\pi, 0, \pi)}(>0)$ and $G_{\|(0,0,0)}(<0)$ which are quite different from those obtained in (a) for weaker $V_{\perp}^{\mathrm{b}}$; SC $f$ fluctuations are then dominant, as seen from eq. (37d). The spin gap vanishes since the combination of couplings given by eq. (39) remains positive. Thus if $\Delta_{\sigma}=0$, the dominant contribution to the SC coupling comes from density fluctuations for which the relevant coupling with parallel spins, namely $G_{\|(\pi, 0, \pi)}(>0)$, is connected to charge fluctuations. It is worth noting that long wave length spin fluctuations also promote the $\mathrm{SC} f$ state since that $G_{\|(0,0,0)}<0$ strengthens $\operatorname{SC} f$ correlations and also the uniform spin susceptibility [eq. (41)], as it will be discussed later. The $\mathrm{CDW}$ fluctuations are well developed at temperatures just above the region where $\mathrm{SC} f$ is dominant [Fig. 9 (b)]. At these temperatures, the CDW response function is nearly the same as that of SDW, implying a coexistence of spin and charge fluctuations for intermediate $V_{\perp}^{\mathrm{b}}$. We finally note that the dominance of SC $f$ state is obtained in the presence of nesting deviations $\left(t_{2}^{*} \neq 0\right)$, which suppresses the divergence of CDW in the low temperature limit.

For $V_{\perp}^{\mathrm{b}}=1.0$ [Fig. 8 (c)], the relevant couplings are $G_{\|(\pi, 0,0)}(>0), G_{\|(\pi, 0, \pi)}(>0), G_{1 \perp(\pi, 0,0)}(<0)$, and $G_{1 \perp(\pi, 0, \pi)}(<0)$, which lead to a CDW state as seen from eq. 


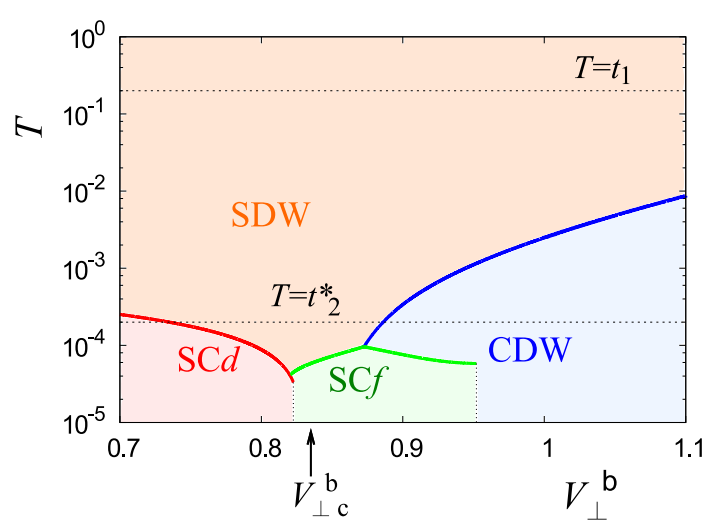

Fig. 10. (Color online) The $T-V_{\perp}^{\mathrm{b}}$ "phase diagram" with $t_{2}^{*} / t_{1}=0.001$ and $h=0.0$

(37a). The behavior of $G_{\|(\pi, 0, \pi)}(>0)$ is similar to that of (b), and then a similarity of CDW and SC $f$ response functions is expected. However, the nature of the spin gap as induced by $G_{1 \perp(\pi, 0,0)}(<0)$ differs. For large $V_{\perp}^{\mathrm{b}}$, it is expected that the dominant state is $\mathrm{CDW}$, whereas $\mathrm{SC} f$ is sub-dominant. Here the difference in sign for $G_{1 \perp(\pi, 0, \pi)}$ in comparison to the case (a) is essential to the predominance of either SCd or CDW.

The dominant states are summarized in the $V_{\perp}^{\mathrm{b}}-T$ phase diagram of Fig. 10. This phase diagram shows some similarity with the one of Fig. 6 in the sense that the SDW state is found at high temperature, namely for $T \gtrsim t_{2}^{*}$. However, for $T \lesssim t_{2}^{*}$, the SC $f$ state comes in between the regions of the SCd and CDW states. In the zone where $T \lesssim t_{2}^{*}$ and $V_{\perp}^{\mathrm{b}} \gtrsim V_{\perp \mathrm{c}}^{\mathrm{b}}$, the $\mathrm{CDW}$ state is dominant and the $\mathrm{SC} f$ state is sub-dominant, but the CDW state is suppressed and the SC $f$ state becomes dominant for $t_{2}^{*} \neq 0-$ a result well explained by the fact that nesting deviations have a detrimental influence primarily on density-wave correlations. The effect of $t_{2}^{*}$, when $V_{\perp}^{\mathrm{b}}<V_{\perp c}^{\mathrm{b}}$, is small, because the $\mathrm{SC} d$ state is less affected by nesting deviations in that region.

At this point we would like to comment on the behavior of the uniform magnetic susceptibility, $\chi_{s}$, given by eq. (41). In Fig. 11, the temperature dependence of this quantity is shown for the $V_{\perp}^{\mathrm{b}}=0.7,0.88$, and 1.0 cases considered above, which correspond to the $\mathrm{SC} d, \mathrm{SC} f$, and CDW states, respectively. It should be noticed that $\chi_{s}$ increases when the system is entering in the SC $f$ state, indicating an enhancement of long wave

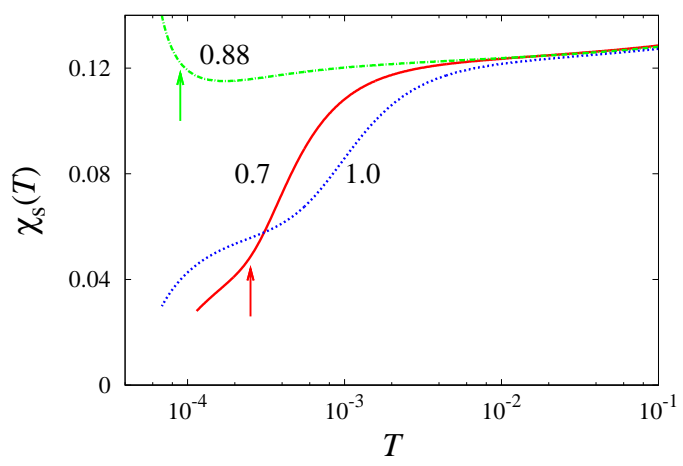

Fig. 11. (Color online) Temperature dependence of the uniform susceptibility for $V_{\perp}^{\mathrm{b}}=0.7,0.88$, and 1.0 , where the parameters are the same as Figs. 9 and 8 . The arrow denotes the temperature at which the SC state becomes dominant with decreasing temperature.

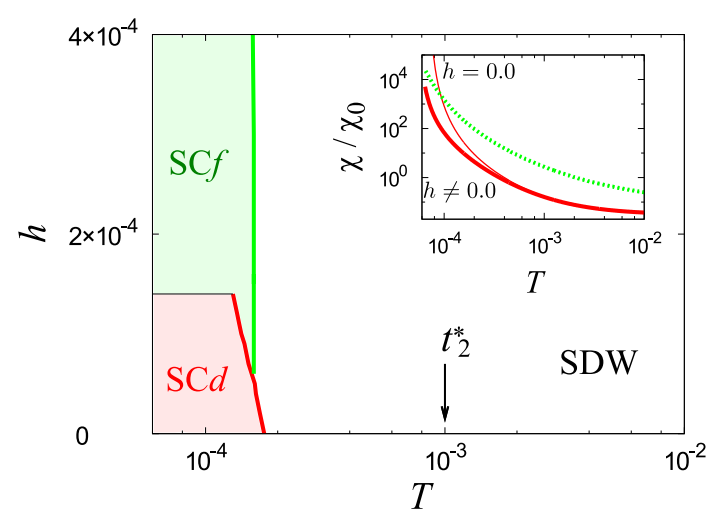

Fig. 12. (Color online) Phase diagram of the most dominant states in the $T$ - $h$ plane with $V_{\perp}^{\mathrm{b}}=0.78$ and $t_{2}^{*} / t_{1}=0.005$. In the inset, the temperature dependence of the SCd (solid line) and SC $f$ (dotted line) response functions is shown where the thin (bold) solid line corresponds to the case for $h=0(0.0002)$.

length spin correlations. This contrasts with the cases where SC $d$ and CDW states prevail and $\chi_{s}$ decreases rapidly due to the formation of a spin gap.

Now we study the effect of a magnetic field on the SCd state for the intermediate interchain interaction, namely in the region where the sub-dominant $\mathrm{SC} f$ state is close to SC $d$. Figure 12 shows the $T$ vs $h$ phase diagram for the most dominant states at $V_{\perp}^{\mathrm{b}}=0.78$. At low temperature, there is a crossover from SC $d$ to the SC $f$ state due to the suppression of the spin gap with $h$. In the inset, the temperature dependence of the response functions for SC $d$ and SC $f$ are shown at finite $(h=$ 0.0002) and zero magnetic field. The SCd correlations are strongly suppressed by $h$, while the magnetic field has essentially no influence on $\mathrm{SC} f$ correlations.

\section{Summary and Discussion}

We have examined by the two-loop RG method the singlet SC $d$ and the triplet SC $f$ superconducting states in the framework of a 4-patch model with nesting deviations. The triplet SC $f$ state, which is absent in the model for intrachain interactions only, is found to develop from the combined effect of interchain repulsive interactions and nesting deviations.

It is of importance to return to the mechanism of formation of the superconducting state and examine the scattering processes that are pertinent to this state. This is shown in Fig. 13, where the symbol \pm in the $k_{\|}-k$ plane denotes the sign of the superconducting gap function for the $\mathrm{SC} d$ [i.e., $\cos k$ in eq. (33c) (a)] and SC $f$ [i.e., $\sin k_{\|} \cos k$ in eq. (33c) (b)] phases. The scattering processes for parallel and anti-parallel spins are described by a dashed arrow, while the long continuous arrows stand for the nesting vector. The sign of the renormalized coupling constants are also stated in each case considered. A sign change of the gap function following the scattering occurs for positive renormalized coupling constants, while the sign remains the same for attractive ones. Thus all the scattering processes shown in Fig. 13 gain the energy.

Figure 13 (a) depicts scattering processes with anti-parallel spins leading to the $\mathrm{SC} d$ state. The interaction with interchain momentum transfer $\pi$, i.e., the interband scatterings, give rise to the repulsive couplings among which $G_{2 \perp(\pi, 0, \pi)}$ is the dominant contribution and $G_{1 \perp(\pi, 0, \pi)}$ also becomes relevant, leading to the growth of spin fluctuations as explained after eq. 
(a)
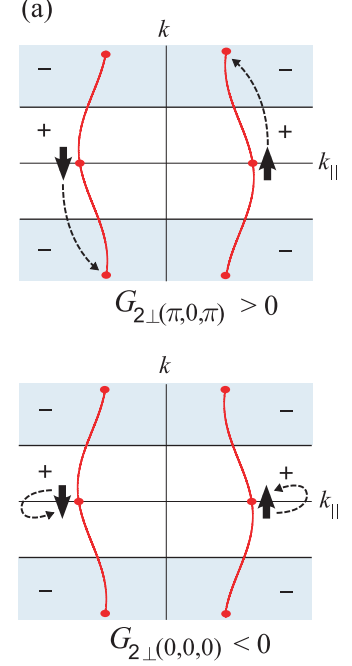
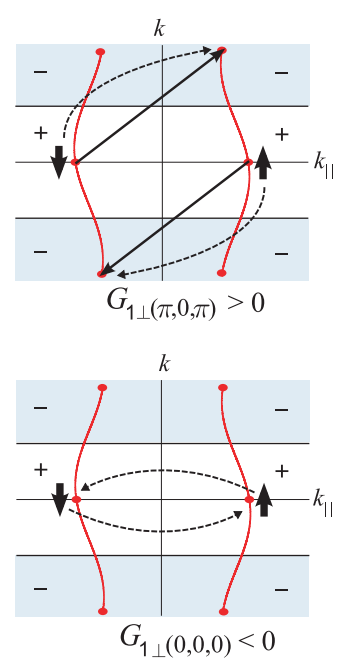

(b)
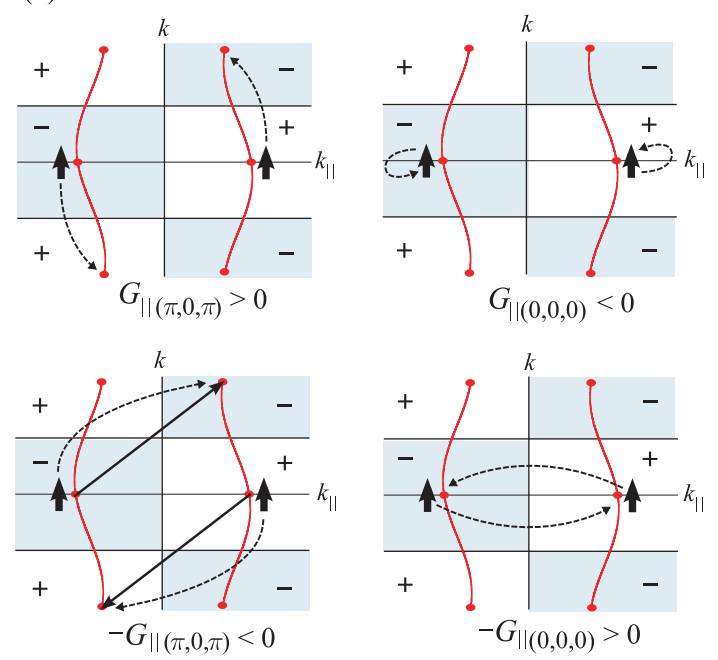

Fig. 13. (Color online) Scattering processes giving rise to the $\mathrm{SC} d$ state (a) and SC $f$ state (b). The Fermi surface is given by the line connecting the Fermi points (closed circle).

(38). The sign of the gap function changes in these scattering process. For the couplings $G_{2 \perp(0,0,0)}$ and $G_{1 \perp(0,0,0)}$, which become attractive through the renormalization, the sign of the gap function remains the same in the scattering process.

We now turn to the $\mathrm{SC} f$ state, where the relation between the sign change of the gap function and the forward scattering process with parallel spins is shown in Fig. 13 (b) (upper panel). For the repulsive $G_{\|(\pi, 0, \pi)}$, the gap function is opposite in sign, whereas the sign is the same for the attractive $G_{\|(0,0,0)}$. The backward scattering shown in the lower (b) panel also favors the SC $f$ state, since the signs of the respective coupling constants, $-G_{\|(\pi, 0, \pi)}<0$ and $-G_{\|(0,0,0)}>0$, are consistent with the change of the sign in the gap function.

We now comment on the crossover from the $\mathrm{SC} d$ to $\mathrm{SC} f$ states as a function of $V_{\perp}^{\mathrm{b}}$. The $V_{\perp}^{\mathrm{b}}$ dependence of relevant coupling constants at low temperature $\left(T=10^{-3}\right)$ is shown in Figs. 14 (a) and (b), which correspond to the $\mathrm{SC} d$ and SC $f$ cases, respectively. For the SC $d$ state, the coupling $G_{1 \perp(0,0,0)}$ evolves from negative to positive values, suggesting a vanishing spin gap. The resultant reduction of the $\mathrm{SC} d$ state by $V_{\perp}^{\mathrm{b}}$ is reasonable because the interchain spin singlet state is destroyed by the formation of CDW state for moderate $V_{\perp}^{\mathrm{b}}$. With increasing $V_{\perp}^{\mathrm{b}}$, the repulsive interactions $G_{2 \perp(\pi, 0, \pi)}$ and $G_{1 \perp(\pi, 0, \pi)}$ decrease and the amplitude of the SC $d$ correlation is reduced.

In Fig. 14 (b), the coupling $G_{\|(\pi, 0, \pi)}$ increases from zero, while the coupling $G_{\|(0,0,0)}$ decreases to negative value. Negative $G_{\|(0,0,0)}$ indicates the absence of spin gap. These features yield in turn the development of the SC $f$ state, as a consequence of charge fluctuations and nesting deviations. From Fig. 14 (b), the $V_{\perp}^{\mathrm{b}}$ interval, where $G_{\sigma+}>0$, suggests that the spin gap vanishes for $V_{\perp}^{\mathrm{b}} \sim V_{\perp c}^{\mathrm{b}}$. However, it is found that the $\mathrm{SC} f$ state moves to the CDW state by noting that $G_{\sigma+}>0$ for $V_{\perp}^{\mathrm{b}}=0.88$, and $G_{\sigma+}<0$ for $V_{\perp}^{\mathrm{b}}=0.92$.

Finally, we comment on the effect of $V_{\perp}^{\mathrm{b}}$ on the SDW state. Within the conventional treatment of RPA, ${ }^{14-16)}$ the interference effect between the scattering at different transverse momenta is neglected. For example, in eq. (9b), which is relevant to the SDW state, the onsite-repulsion $U$ is retained but the effect of $V_{\perp}^{\mathrm{b}}$ is strongly reduced due to the summa- (a)

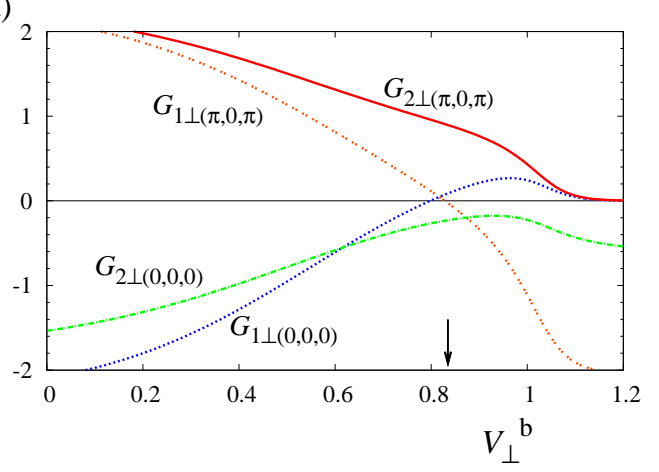

(b)

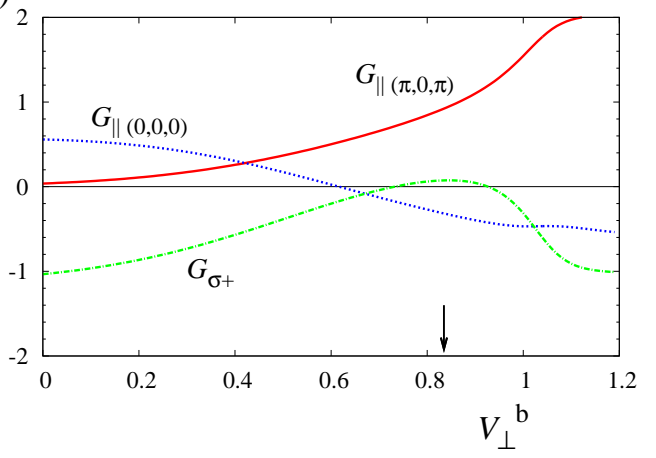

Fig. 14. (Color online) The $V_{\perp}^{\mathrm{b}}$ dependence of the renormalized coupling constants at $T=10^{-3}$ for the $\mathrm{SC} d$ (a) and $\mathrm{SC} f$ (b) states, and for $G_{\sigma+}$. Here $t_{2}^{*}=0.001 t_{1}$ and $h=0.0$. The initial values for $G_{1 \perp(0,0,0)}, G_{1 \perp(\pi, 0, \pi)}$, $G_{2 \perp(0,0,0)}, G_{2 \perp(\pi, 0, \pi)}, G_{\|(0,0,0)}$ and $G_{\|(\pi, 0, \pi)}$, are respectively given by $0.45,0.45,0.45,0.45,0.00$ and 0.00 for $V_{\perp}^{\mathrm{b}}=0$ and $0.72,0.18,0.45,0.45$, -0.27 and 0.27 for $V_{\perp}^{\mathrm{b}}=1.2$. The arrow represents $V_{\perp c}^{\mathrm{b}}$

tion of $k_{1}= \pm \pi$ and $k_{2}= \pm \pi$. However, the present RG method shows a clear effect of $V_{\perp}^{\mathrm{b}}$ on SDW as illustrated by the strength of density-wave correlations in the $V_{\perp}^{\mathrm{b}}-U$ plane at fixed $T$ (Fig. 15). The SDW and CDW regions are defined by the corresponding response functions that become larger than the bare value $\chi_{0}$ at $l=0$ by a factor greater than $10^{6}$. The domain that separates the two regions shows a much reduced amplitude of the density-wave response functions, while its area reduces by the decrease of temperature. The region for 


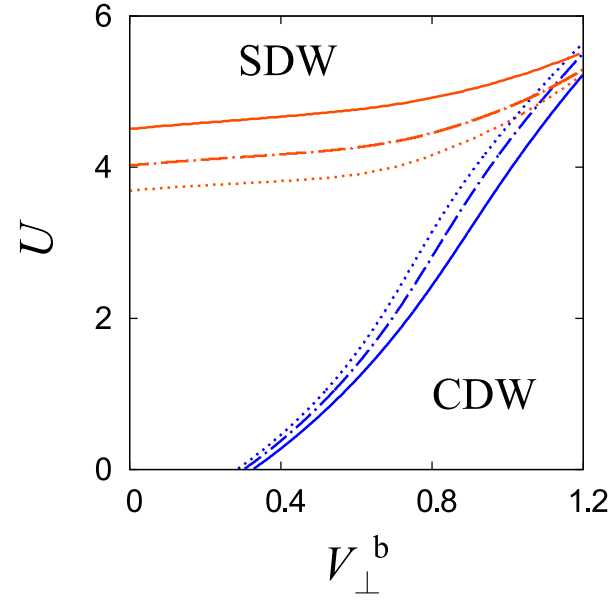

Fig. 15. (Color online) The regions of SDW and CDW dominant correlations in the $V_{\perp}^{\mathrm{b}}-U$ plane defined by the condition $\chi_{\mathrm{SDW}}\left(\chi_{\mathrm{CDW}}\right)>10^{6} \chi_{0}$ at the fixed temperatures $T=2 \times 10^{-4}$ (dashed line), $4 \times 10^{-4}$ (dash-dotted) and $8 \times 10^{-4}$ (solid line). Here $t_{2}^{*}=2 \times 10^{-4}$. In the remaining in between region $\chi_{\mathrm{SDW}}\left(\chi_{\mathrm{CDW}}\right)<10^{6} \chi_{0}$.

SDW is suppressed with increasing $V_{\perp}^{\mathrm{b}}$ due to the increase of the charge fluctuations.

\section{Acknowledgments}

The present research was partially supported by Grant-inAid for Scientific Research on Innovative Areas 20110002.

\section{Appendix: Two-loop level renormalization group}

The renormalization of the interchain hopping $t_{1}$ is given by $^{24)}$

$$
\frac{d}{d l} t_{1}(l)=t_{1}(l)-\frac{1}{2 N_{\perp}^{2}} \sum_{q, k, k^{\prime}} G_{\Sigma_{n}\left(q, k, k^{\prime}\right)} J_{0\left(q, k, k^{\prime}\right)},
$$

where the second term of r.h.s. comes from interactions. Such an effect is negligibly small for the quarter-filled band although the strong effect is expected due to the Umklapp scattering for the half-filled band, e.g., the confinement of the interchain hopping. ${ }^{26,27)}$

We show the results at the two-loop level in eq. (30). Applying the calculations already obtained at perfect nesting and half-filling ${ }^{24)}$ to the case of the nesting deviation at quarter-filling, the self-energy and vertex corrections are $\Xi_{\Sigma\left(q, k_{1}, k_{2}, q^{\prime}, k^{\prime}\right)}^{(2)}, \Xi_{v\left(q, k_{1}, k_{2}, q^{\prime}, k^{\prime}\right)}^{(2)}$, which read

$$
\begin{aligned}
& \Xi_{\Sigma\left(q, k_{1}, k_{2}, q^{\prime}, k^{\prime}\right)}^{(2)}=G_{\Sigma_{n}\left(q^{\prime}, k_{1}, k^{\prime}\right)}^{2} J_{1\left(q^{\prime}, k_{1}, k^{\prime}\right)} \\
& \quad+G_{\Sigma_{n}\left(q^{\prime}, k_{2}, k^{\prime}\right)}^{2} J_{1\left(q^{\prime}, k_{2}, k^{\prime}\right)}+G_{\Sigma_{n}\left(q^{\prime},-k_{1}+q, k^{\prime}\right)}^{2} J_{1\left(q^{\prime},-k_{1}+q, k^{\prime}\right)} \\
& \quad+G_{\Sigma_{n}\left(q^{\prime},-k_{2}+q, k^{\prime}\right)}^{2} J_{1\left(q^{\prime},-k_{2}+q, k^{\prime}\right)},
\end{aligned}
$$

$$
\begin{aligned}
& \Xi_{1 \perp\left(q, k_{1}, k_{2}, q^{\prime}, k^{\prime}\right)}^{(2)}=J_{2\left(q+k^{\prime} ; k_{1}, k_{2} ; k^{\prime}, k^{\prime}-q^{\prime}\right)} \\
& \quad \times\left[G_{1 \perp\left(q+q^{\prime}, k_{1}, k_{2}\right)} G_{2 \perp\left(q-k_{2}+k^{\prime}, k^{\prime}-q^{\prime}, k^{\prime}\right)} G_{\|\left(q-k_{1}+k^{\prime}, k^{\prime}, k^{\prime}-q^{\prime}\right)}\right. \\
& \left.\quad+G_{1 \perp\left(q+q^{\prime}, k_{1}, k_{2}\right)} G_{\|\left(q-k_{2}+k^{\prime}, k^{\prime}-q^{\prime}, k^{\prime}\right)} G_{2 \perp\left(q-k_{1}+k^{\prime}, k^{\prime}, k^{\prime}-q^{\prime}\right)}\right] \\
& \quad+J_{2\left(-k^{\prime} ;-k_{1},-k_{2} ; \pi-k^{\prime}, \pi-k^{\prime}+q^{\prime}\right)} \\
& \quad \times\left[G_{2 \perp\left(k_{1}-k^{\prime}, k_{1}, k_{1}-q^{\prime}\right)} G_{\|\left(k_{2}-k^{\prime}, k_{2}-q^{\prime}, k_{2}\right)} G_{1 \perp\left(q-q^{\prime}, k_{1}-q^{\prime}, k_{2}-q^{\prime}\right)}\right.
\end{aligned}
$$

$$
\left.+G_{\|\left(k_{1}-k^{\prime}, k_{1}, k_{1}-q^{\prime}\right)} G_{2 \perp\left(k_{2}-k^{\prime}, k_{2}-q^{\prime}, k_{2}\right)} G_{1 \perp\left(q-q^{\prime}, k_{1}-q^{\prime}, k_{2}-q^{\prime}\right)}\right],
$$

$$
\begin{aligned}
& \Xi_{2 \perp\left(q, k_{1}, k_{2}, q^{\prime}, k^{\prime}\right)}^{(2)}=J_{2\left(q+k^{\prime} ; k_{1}, k_{2} ; k^{\prime}, k^{\prime}-q^{\prime}\right)} \\
& \quad \times\left[G_{2 \perp\left(q+q^{\prime}, k_{1}, k_{2}\right)} G_{2 \perp\left(q-k_{2}+k^{\prime}, k^{\prime}-q^{\prime}, k^{\prime}\right)} G_{2 \perp\left(q-k_{1}+k^{\prime}, k^{\prime}, k^{\prime}-q^{\prime}\right)}\right. \\
& \quad+G_{2 \perp\left(q+q^{\prime}, k_{1}, k_{2}\right)} G_{\|\left(q-k_{2}+k^{\prime}, k^{\prime}-q^{\prime}, k^{\prime}\right)} G_{\|\left(q-k_{1}+k^{\prime}, k^{\prime}, k^{\prime}-q^{\prime}\right)} \\
& \left.\quad+G_{\|\left(q+q^{\prime}, k_{1}, k_{2}\right)} G_{1 \perp\left(q-k_{2}+k^{\prime}, k^{\prime}-q^{\prime}, k^{\prime}\right)} G_{1 \perp\left(q-k_{1}+k^{\prime}, k^{\prime}, k^{\prime}-q^{\prime}\right)}\right] \\
& \quad+J_{2\left(-k^{\prime} ;-k_{1},-k_{2} ; \pi-k^{\prime}, \pi-k^{\prime}+q^{\prime}\right)} \\
& \quad \times\left[G_{2 \perp\left(k_{1}-k^{\prime}, k_{1}, k_{1}-q^{\prime}\right)} G_{2 \perp\left(k_{2}-k^{\prime}, k_{2}-q^{\prime}, k_{2}\right)} G_{2 \perp\left(q-q^{\prime}, k_{1}-q^{\prime}, k_{2}-q^{\prime}\right)}\right. \\
& \quad+G_{\|\left(k_{1}-k^{\prime}, k_{1}, k_{1}-q^{\prime}\right)} G_{\|\left(k_{2}-k^{\prime}, k_{2}-q^{\prime}, k_{2}\right)} G_{2 \perp\left(q-q^{\prime}, k_{1}-q^{\prime}, k_{2}-q^{\prime}\right)} \\
& \left.\quad+G_{1 \perp\left(k_{1}-k^{\prime}, k_{1}, k_{1}-q^{\prime}\right)} G_{1 \perp\left(k_{2}-k^{\prime}, k_{2}-q^{\prime}, k_{2}\right)} G_{\|\left(q-q^{\prime}, k_{1}-q^{\prime}, k_{2}-q^{\prime}\right)}\right],
\end{aligned}
$$

$$
\begin{aligned}
& \Xi_{\|\left(q, k_{1}, k_{2}, q^{\prime}, k^{\prime}\right)}^{(2)}=J_{2\left(q+k^{\prime} ; k_{1}, k_{2} ; k^{\prime}, k^{\prime}-q^{\prime}\right)} \\
& \quad \times\left[G_{\|\left(q+q^{\prime}, k_{1}, k_{2}\right)} G_{2 \perp\left(q-k_{2}+k^{\prime}, k^{\prime}-q^{\prime}, k^{\prime}\right)} G_{2 \perp\left(q-k_{1}+k^{\prime}, k^{\prime}, k^{\prime}-q^{\prime}\right)}\right. \\
& \quad+G_{\|\left(q+q^{\prime}, k_{1}, k_{2}\right)} G_{\|\left(q-k_{2}+k^{\prime}, k^{\prime}-q^{\prime}, k^{\prime}\right)} G_{\|\left(q-k_{1}+k^{\prime}, k^{\prime}, k^{\prime}-q^{\prime}\right)} \\
& \left.\quad+G_{2 \perp\left(q+q^{\prime}, k_{1}, k_{2}\right)} G_{1 \perp\left(q-k_{2}+k^{\prime}, k^{\prime}-q^{\prime}, k^{\prime}\right)} G_{1 \perp\left(q-k_{1}+k^{\prime}, k^{\prime}, k^{\prime}-q^{\prime}\right)}\right] \\
& \quad+J_{2\left(-k^{\prime} ;-k_{1},-k_{2} ; \pi-k^{\prime}, \pi-k^{\prime}+q^{\prime}\right)} \\
& \quad \times\left[G_{2 \perp\left(k_{1}-k^{\prime}, k_{1}, k_{1}-q^{\prime}\right)} G_{2 \perp\left(k_{2}-k^{\prime}, k_{2}-q^{\prime}, k_{2}\right)} G_{\|\left(q-q^{\prime}, k_{1}-q^{\prime}, k_{2}-q^{\prime}\right)}\right. \\
& \quad+G_{\|\left(k_{1}-k^{\prime}, k_{1}, k_{1}-q^{\prime}\right)} G_{\|\left(k_{2}-k^{\prime}, k_{2}-q^{\prime}, k_{2}\right)} G_{\|\left(q-q^{\prime}, k_{1}-q^{\prime}, k_{2}-q^{\prime}\right)} \\
& \left.+G_{1 \perp\left(k_{1}-k^{\prime}, k_{1}, k_{1}-q^{\prime}\right)} G_{1 \perp\left(k_{2}-k^{\prime}, k_{2}-q^{\prime}, k_{2}\right)} G_{2 \perp\left(q-q^{\prime}, k_{1}-q^{\prime}, k_{2}-q^{\prime}\right)}\right],
\end{aligned}
$$

where $G_{\Sigma_{n}\left(q, k, k^{\prime}\right)}^{2}$ is given by

$$
G_{\Sigma_{n}\left(q, k, k^{\prime}\right)}^{2}=\sum_{v=1 \perp, 2 \perp, \|} G_{v}^{2}\left(q, k, k^{\prime}\right)
$$

and $J_{0\left(q, k, k^{\prime}\right)}, J_{1\left(q, k, k^{\prime}\right)}$ are given as follows. For $\left|Y_{q, k, k^{\prime}, 0}^{\mathrm{P}}(l)\right|<$ $E$,

$$
\begin{aligned}
& J_{0\left(q, k, k^{\prime}\right)}=2 E \ln \left[\frac{4 E+Y_{q, k, k^{\prime}, 0}^{\mathrm{P}}(l)}{4 E-Y_{q, k, k^{\prime}, 0}^{\mathrm{P}}(l)}\right] \\
& J_{1\left(q, k, k^{\prime}\right)}=\frac{16 E^{2}}{16 E^{2}-\left(Y_{q, k, k^{\prime}, 0}^{\mathrm{P}}(l)\right)^{2}} .
\end{aligned}
$$

For $\left|Y_{q, k, k^{\prime}}^{\mathrm{P}}(l)\right|>E$,

$$
\begin{aligned}
& J_{0\left(q, k, k^{\prime}\right)}=2 E \ln \left[\frac{4 E+\left|Y_{q, k, k^{\prime}, 0}^{\mathrm{P}}(l)\right|}{4 E+\left|Y_{q, k, k^{\prime}, 0}^{\mathrm{P}}(l)\right|}\right] \operatorname{sgn}\left(Y_{q, k, k^{\prime}, 0}^{\mathrm{P}}(l)\right), \\
& J_{1\left(q, k, k^{\prime}\right)}=\frac{2 E}{4 E+\left|Y_{q, k, k^{\prime}, 0}^{\mathrm{P}}(l)\right|}+\frac{2 E}{2 E+\left|Y_{q, k, k^{\prime}, 0}^{\mathrm{P}}(l)\right|} .
\end{aligned}
$$

The quantity $J_{2\left(q+k^{\prime \prime} ; k_{1}, k_{2} ; k^{\prime}, k^{\prime \prime}\right)}$ is also given by

$$
J_{2\left(q+k^{\prime \prime} ; k_{1}, k_{2} ; k^{\prime}, k^{\prime \prime}\right)}=\frac{1}{2}\left[J_{1\left(q+k^{\prime \prime}-k_{1}, k^{\prime}, k^{\prime \prime}\right)}+J_{1\left(q+k^{\prime \prime}-k_{2}, k^{\prime}, k^{\prime \prime}\right)}\right] .
$$

In the present calculations carried out at the two-loop level, we treated nesting deviations as the dominant effect. The influence of magnetic field at that level remains to be examined. This will be the subject of a separate publication. 
1) G. Grüner: Density Waves in Solids (Addison Wesley, Massachusetts and Tokyo, 2007).

2) K. Kuroki: J. Phys. Soc. Jpn. 75 (2006) 051013.

3) I. J. Lee, M. J. Naughton, G. M. Danner, and P. M. Chaikin: Phys. Rev. Lett. 78 (1997) 3555.

4) W. Zhang and C.A.R. Sá de Melo: Adv. Phys. 56 (2007) 545.

5) J. Shinagawa, Y. Kurosaki, F. Zhang, C. Parker, S. E. Brown, D. Jérome, K. Bechgaard, and J. B. Christensen: Phys. Rev. Lett. 98 (2007) 147002.

6) S. Yonezawa, S. Kusaba, Y. Maeno, P. Auban-Senzier, C. Pasquier, K. Bechgaard, and D. Jérome: Phys. Rev. Lett. 100 (2008) 117002.

7) P. Wzietek, F. Creuzet, C. Bourbonnais, D. Jérome, K. Bechgaard, and P. Batail: J. Phys. I 3 (1993) 171.

8) H. Shimahara: J. Phys. Soc. Jpn. 69 (2000) 1966.

9) H. Shimahara: Phys. Rev. B 62 (2000) 3524.

10) N. Belmechri, G. Abramovici, M. Heritier, S. Haddad, and S. CharfiKaddour: Eur. Phys. Lett. 80 (2007) 37004.

11) N. Belmechri, G. Abramovici, and M. Heritier: Eur. Phys. Lett. 82 (2008) 47009.

12) H. Aizawa, K. Kuroki, and Y. Tanaka: Phys. Rev. B 77 (2008) 144513.

13) H. Aizawa, K. Kuroki, T. Yokoyama, and Y. Tanaka: Phys. Rev. Lett. 102 (2009) 016403.

14) D.J. Scalapino, E. Loh, Jr., and J.E. Hirsch: Phys. Rev. B 35 (1987) 6694.

15) H. Shimahara: J. Phys. Soc. Jpn. 58 (1989) 1735.

16) Y. Tanaka and K. Kuroki: Phys. Rev. B 70 (2004) 060502.

17) J. Sólyom: Adv. Phys. 28 (1979) 201.
18) C. Bourbonnais, B. Guay, and R. Wortis: in Theoretical Methods for Strongly Correlated Electrons, edited by D. Seńećhal, A.M. Tremblay, and C. Bourbonnais (Springer, New York, 2003) p. 77.

19) K. Penc and J. Sólyom: Phys. Rev. B 47 (1993) 6273.

20) G. Montambaux, M. Héritier, and P. Lederer: Phys. Rev. B 33 (1986) 7777.

21) R. Duprat and C. Bourbonnais: Eur. Phys. J. B. 21 (2001) 219.

22) Y. Fuseya and Y. Suzumura: J. Phys. Soc. Jpn. 74 (2005) 1263.

23) J. C. Nickel, R. Duprat, C. Bourbonnais, and N. Dupuis: Phys. Rev. Lett. 95 (2005) 247001.

24) M. Tsuchiizu: Phys. Rev. B. 74 (2006) 155109.

25) G. Abramovici, J. C. Nickel, and M. Héritier: Phys. Rev. B. 72 (2005) 045120.

26) Y. Suzumura, M. Tsuchiizu, and G. Grüner: Phys. Rev. B 57 (1998) R15040.

27) M. Tsuchiizu and Y. Suzumura: Phys. Rev. B 59 (1999) 12326.

28) V.J. Emery, R. Bruinsma, and S. Barisić: Phys. Rev. Lett. 48 (1982) 1039.

29) M. Tsuchiizu and Y. Suzumura: Phys. Rev. B 72 (2005) 075121.

30) Y. Fuseya, M. Tsuchiizu, Y. Suzumura, and C. Bourbonnais: J. Phys. Soc. Jpn. 74 (2005) 3159.

31) Y. Fuseya, M. Tsuchiizu, Y. Suzumura, and C. Bourbonnais: J. Phys. Soc. Jpn. 76 (2007) 014709.

32) M. Fabrizio: Phys. Rev. B. 48 (1993) 15838. 